\title{
A COMPARATIVE ASTROCHEMICAL STUDY OF THE HIGH-MASS PROTOSTELLAR OBJECTS NGC 7538 IRS 9 AND IRS 1
}

\author{
John C. Barentine and John H. LaCY \\ Department of Astronomy, The University of Texas at Austin, 1 University Station, C1400, Austin, TX 78712-0259, USA \\ Received 2012 April 20; accepted 2012 August 8; published 2012 September 10
}

\begin{abstract}
We report the results of a spectroscopic study of the high-mass protostellar object NGC 7538 IRS 9 and compare our observations to published data on the nearby object NGC 7538 IRS 1. Both objects originated in the same molecular cloud and appear to be at different points in their evolutionary histories, offering an unusual opportunity to study the temporal evolution of envelope chemistry in objects sharing a presumably identical starting composition. Observations were made with the Texas Echelon Cross Echelle Spectrograph, a sensitive, high spectral resolution $(R=\lambda / \Delta \lambda \simeq 100,000)$ mid-infrared grating spectrometer. Forty-six individual lines in vibrational modes of the molecules $\mathrm{C}_{2} \mathrm{H}_{2}, \mathrm{CH}_{4}, \mathrm{HCN}, \mathrm{NH}_{3}$, and $\mathrm{CO}$ were detected, including two isotopologues $\left({ }^{13} \mathrm{CO},{ }^{12} \mathrm{C}^{18} \mathrm{O}\right)$ and one combination mode $\left(v_{4}+v_{5} \mathrm{C}_{2} \mathrm{H}_{2}\right)$. Fitting synthetic spectra to the data yielded the Doppler shift, excitation temperature, Doppler $b$ parameter, column density, and covering factor for each molecule observed; we also computed column density upper limits for lines and species not detected, such as HNCO and OCS. We find differences among spectra of the two objects likely attributable to their differing radiation and thermal environments. Temperatures and column densities for the two objects are generally consistent, while the larger line widths toward IRS 9 result in less saturated lines than those toward IRS 1. Finally, we compute an upper limit on the size of the continuum-emitting region $(\sim 2000 \mathrm{AU})$ and use this constraint and our spectroscopy results to construct a schematic model of IRS 9.
\end{abstract}

Key words: ISM: individual objects (NGC 7538 IRS 9, NGC 7538 IRS 1) - stars: formation - stars: pre-main sequence - techniques: spectroscopic

\section{INTRODUCTION}

Details of the pre-main-sequence (pre-MS) life cycles of low-mass stars such as the Sun $\left(M \sim 1 M_{\odot}\right)$ have been gleaned from decades of research; see McKee \& Ostriker (2007) for a recent review. This is thanks in part to the relative amenability of low-mass protostellar objects to study. However, details of high-mass $\left(M>8 M_{\odot}\right)$ star formation are not as well understood at present, in part because high-mass stars are not generally forming near the solar neighborhood and remain deeply embedded during their pre-MS evolution. An important unresolved issue is whether high-mass stars form in a manner resembling a scaled-up version of low-mass star formation (Keto \& Zhang 2010; Johnston et al. 2011) or through other mechanisms uncharacteristic of the low-mass case such as collisions/mergers (Bonnell et al. 1998; Bonnell \& Bate 2005; Bally \& Zinnecker 2005) and competitive accretion in clusters (Bonnell et al. 2001, 2004). Clearly, the environments in which high-mass star formation takes place seem to influence the formation process itself. Massive stars are most often seen to form in large groups ("OB associations") near the edges of dense clumps in giant molecular clouds (GMCs; Zinnecker \& Yorke 2007). Elmegreen \& Lada (1977) first suggested that spatially distinct subgroups of stars within OB associations trigger waves of self-propagating star formation in GMCs via shock/ionization fronts that progress from the outside in. This process may account for observations of $\mathrm{OB}$ subgroups that lie in linear arrangements along the Galactic plane, showing a monotonically increasing sequence of ages (Blaauw 1964, 1991). Rapid improvements in both observational capabilities and theoretical sophistication in recent years are advancing our knowledge of the circumstances of massive star formation and enabling these fundamental questions to be addressed.
The origin of these stars is of particular interest due to their role in the Galactic ecosystem. In addition to serving as the principal sites of heavy element nucleosynthesis, the violent end of massive stars in supernovae injects considerable mechanical energy into the interstellar medium (ISM) while enriching it with metals (Arnett 1996). The debris are reconstituted in molecular clouds from which new stars form, often accompanied by circumstellar disks (Jiang et al. 2008). These disks are evidently the source of accretion onto high-mass stars as they approach the MS in the Hertzsprung-Russell diagram and ignite hydrogen burning, then continue to accrete their final masses, moving parallel to the MS (Zinnecker \& Yorke 2007). Relatively little is known about the structure and chemical composition of massive protostellar envelopes, and even less about how they evolve in time. Ready comparison with envelope models for low-mass objects is instructive but only of limited use, in part due to the radically different radiation environment in the high-mass case. The composition and structure of the envelope are of interest as they determine the final mass of the star through an accretion mechanism whose details are still unclear. Piecing together the components of protostellar envelopes is complicated by the observation that most objects are deeply embedded, particularly early in the formation process. Spectroscopy of molecules is useful in delineating physical structures at high extinction where other methods are limited. Pure rotational transitions of molecules in the millimeter probe a sufficiently low-energy regime but such observations are typically of low spatial resolution. Better resolution is achieved in the infrared where many ro-vibrational molecular lines are located.

We present high-resolution, mid-infrared spectra of a number of molecules toward an embedded infrared source in the NGC 7538 star-forming complex, NGC 7538 IRS 9, and compare the results to a previous study of the nearby source 
NGC 7538 IRS 1 (Knez et al. 2009). Both sources are presumed to harbor high-mass protostellar objects in their interiors. Their proximity to one another suggests that, having formed within the same molecular cloud, the starting chemical abundances for each object should have been substantially similar. A comparison of their current states provides insights about differences in their ages and/or divergence in their individual evolutionary histories.

Weak free-free emission ( $\leqslant 60 \mu \mathrm{Jy}$ at $3.6 \mathrm{~cm}$; Sandell et al. 2005 ) is observed toward IRS 9. Further evidence for the comparatively primitive nature of the envelope of IRS 9 is given by the detection of various ices in its Infrared Space Observatory (ISO) spectrum (Whittet et al. 1996; Gibb et al. 2004). These ices are not seen toward IRS 1, which appears to be in a relatively advanced point in its pre-MS evolution. It is a considerably stronger source at centimeter wavelengths and shows evidence of both ionized and molecular components in a bipolar outflow originating in a disk seen nearly edge-on (Sandell et al. 2009; Qiu et al. 2011). IRS 1 also shows signs of a circumstellar disk and an emerging compact $\mathrm{H}$ II region; it is thought to be a forming late $\mathrm{O}$ star undergoing active accretion, suggesting a more advanced evolutionary state (Lacy \& Jaffe 2007; Beuther et al. 2012). Sandell et al. (2009) further note that the system is heavily accreting $\left(\dot{M} \sim 2 \times 10^{-4} M_{\odot} \mathrm{yr}^{-1}\right.$ ) and that the accretion may be episodic in nature. On the basis of $\mathrm{GHz}$ line observations, Surcis et al. (2011) conclude that the accretion appears to be the result of radial infall from a "torus" thousands of astronomical units (AU) in size, traced by $\mathrm{H}_{2} \mathrm{O}$ and $\mathrm{CH}_{3} \mathrm{OH}$ masers, instead of the Keplerian disk favored by Pestalozzi et al. (2004).

Until recently, estimates of the distance to NGC 7538 placed it at $23.0 \mathrm{kpc}$ (Heyer et al. 2001; Brunt 2003; Foster \& Routledge 2003; Balog et al. 2004; Reid \& Wilson 2005; Kameya 2006; Araya et al. 2007). However, we adopted the distance of Moscadelli et al. (2009), who find a value of $2.65_{-0.11}^{+0.12} \mathrm{kpc}$ from trigonometric parallax measurements. The total luminosity of IRS 9 is about $3.5 \times 10^{4} L_{\odot}$ (Sandell et al. 2005, corrected to the Moscadelli et al. distance) and it is a bright IRAS $12 \mu \mathrm{m}$ source at $\sim 60$ Jy (Beichman et al. 1988). A zero-age main-sequence (ZAMS) star with this luminosity would have a spectral type of B0.5, an effective temperature $T_{\text {eff }}=26200 \mathrm{~K}$ (Panagia 1973), and a mass of $\sim 13 M_{\odot}$ (Ekström et al. 2012). Boogert et al. (2004) suggest the existence of an inner, warm molecular emission region with a radius of $\sim 70 \mathrm{AU}$; the dust temperature at this distance for an object of IRS 9's luminosity would be $\sim 1000 \mathrm{~K}$ depending on the dust mass opacity. Millimeter data show that IRS 9 is a site of active, massive star formation (Sandell et al. 2005) in which young stellar objects are driving a set of bipolar molecular outflows on a dynamical timescale of $\leqslant 20,000$ years. At least one of these outflows appears to be highly energetic. Mitchell \& Hasegawa (1991) report the discovery of an outflow in IRS 9 with a velocity of $110 \mathrm{~km} \mathrm{~s}^{-1}$, which they interpret to have emerged as recently as 1200 years ago, perhaps indicating the beginning of a recent episode of accretion.

Observations of these protostellar objects are broadly consistent with the Elmegreen \& Lada (1977) picture of sequential high-mass star formation, as elaborated on by others in the particular case of NGC 7538 (Werner et al. 1979; Fischer et al. 1980; Dickel et al. 1981). The Campbell \& Thompson (1984) model of NGC 7538 holds that a first generation of massive stars caused the visible $\mathrm{H}$ II region, which expanded and impinged on the neighboring molecular cloud; this compressed the cloud and generated shocks that caused the observed near-infrared fluorescent $\mathrm{H}_{2}$ emission northwest of IRS 1 . In this picture, high-mass star formation is progressing toward the southeast, and IRS 9 belongs to a second wave of star formation as it is placed further along the direction of propagation of the shock front. These objects then represent two snapshots of the evolution of a massive young protostellar object in a particular cloud and are useful in addressing temporal evolution questions.

This paper is organized as follows. In Section 2, we give details concerning how we obtained and reduced the data, followed by a detailed description in Section 3 of the molecular species detected. The data analysis is described in Section 4 with emphasis on spectral modeling, and we give some interpretation to the results in Section 5. We summarize these results and draw some conclusions in Section 6.

\section{OBSERVATIONS AND REDUCTIONS}

We observed NGC 7538 IRS 9 with the Texas Echelon Cross (X) Echelle Spectrograph (TEXES; Lacy et al. 2002) on the Gemini North $8 \mathrm{~m}$ telescope in 2007 October. Similar observations of IRS 1 were carried out with TEXES at the NASA Infrared Telescope Facility (IRTF) $3 \mathrm{~m}$ telescope in 2001 June, 2001 November, 2002 September, 2002 December, and 2005 November. Circumstances for all IRS 9 observations are given in Table 1. IRTF observations of IRS 1 form the basis for the work of Knez et al. (2009). Additional observations of IRS 1 were made at Gemini in 2007 October to supplement previous TEXES data taken at the IRTF.

TEXES is a cross-dispersed grating spectrograph designed to operate at high spectral resolution $(R=75,000-100,000)$ over a range of mid-infrared wavelengths from 5 to $25 \mu \mathrm{m}$. Light diffracts off a coarsely ruled "echelon" grating (Michelson 1898 ) at a large incidence angle with respect to normal at order $n \sim 1500$. The light then diffracts off a cross-dispersion grating and is imaged onto a $256 \times 256$ pixel $\mathrm{Si}$ :As detector as a series of orders. At a given spectral setting between five and ten cross-dispersed orders are imaged onto the detector for a spectral coverage of about $0.5 \%$. The continuity of orders is wavelength dependent; below $11 \mu \mathrm{m}$ order overlap is sufficient to yield continuous coverage over an entire spectral setting. At wavelengths longer than $11 \mu \mathrm{m}$ there are gaps between adjacent orders.

On Gemini the pixel scale of TEXES was $\sim 0^{\prime \prime} 14$ while at the IRTF it was $\sim 0$ ".36. The pixel scales also compare favorably with the diffraction limit at $10 \mu \mathrm{m}$ of each telescope, $0{ }^{\prime \prime} .70$ and $0^{\prime \prime} .26$ at the IRTF and Gemini, respectively. In both cases the seeing disk is, at worst, critically sampled under good seeing conditions. All observations were made at a resolving power $R \simeq 80,000$, or $\Delta v \sim 3-4 \mathrm{~km} \mathrm{~s}^{-1}$ and the spectral coverage at each spectral setting was $\Delta \lambda \approx 0.06 \mu \mathrm{m}$. The spectral sampling of the TEXES array is about $1.0 \mathrm{~km} \mathrm{~s}^{-1}$. Slit widths were 0.5 at Gemini and 1". 4 at the IRTF. The slit length was $\sim 4^{\prime \prime}$ on Gemini and $\sim 10^{\prime \prime}$ on the IRTF, varying with wavelength. We alternated the telescope pointing between two positions on the sky in the slit direction, separated by $\sim 1^{\prime \prime}-5^{\prime \prime}$, during an exposure sequence. Subtraction of adjacent "nod" positions removes the contribution to the signal from night sky emission. The separation of points in the nod, or "nod throw," is determined by the slit length.

To correct for telluric atmospheric absorption, we observed comparison objects whose spectra are close to blackbody over the wavelength range of observation. At wavelengths beyond $\sim 8 \mu \mathrm{m}$, asteroids meet this requirement while providing the 
Table 1

Circumstances of TEXES Observations of NGC 7538 IRS 9

\begin{tabular}{|c|c|c|c|}
\hline UT Date & Telescope & $\begin{array}{l}\text { Wavenumber } \\
\text { Center }\left(\mathrm{cm}^{-1}\right)\end{array}$ & Included Features \\
\hline 2001 Jun 15 & IRTF & $\begin{array}{l}768 \\
780\end{array}$ & $\begin{array}{c}\mathrm{C}_{2} \mathrm{H}_{2} v_{5} R(15,16) \\
{[\mathrm{Ne} \text { II }]\left({ }^{2} P_{1 / 2} \rightarrow{ }^{2} P_{3 / 2}\right), \mathrm{C}_{2} \mathrm{H}_{2} v_{5}} \\
R(21,22), \mathrm{HCN} v_{2} R(22,23)\end{array}$ \\
\hline 2001 Jun 18 & IRTF & 744 & $\mathrm{C}_{2} \mathrm{H}_{2} v_{5} R(5,6), \mathrm{HCN} v_{2} R(10)$ \\
\hline 2001 Jun $28-29$ & IRTF & 734 & $\mathrm{C}_{2} \mathrm{H}_{2} v_{5} R(1), \mathrm{HCN} v_{2} R(6,7)$ \\
\hline 2007 Oct 21 & Gemini & $\begin{array}{r}828 \\
1308\end{array}$ & $\begin{array}{l}\mathrm{HNCO} v_{4} P \text {-branch, }{ }^{14} \mathrm{NH}_{3} v_{2} s P(7, \mathrm{~K}) \\
{ }^{12} \mathrm{CH}_{4} v_{4} R(0),{ }^{13} \mathrm{CH}_{4} v_{4} R(1)\end{array}$ \\
\hline 2007 Oct 25 & Gemini & $\begin{array}{l}930 \\
743\end{array}$ & $\begin{array}{c}{ }^{14} \mathrm{NH}_{3} v_{2} a Q(\mathrm{~J}, \mathrm{~K}) \\
\mathrm{C}_{2} \mathrm{H}_{2} \quad v_{5} R(5,6)\end{array}$ \\
\hline 2007 Oct 27 & Gemini & 2055 & $\begin{array}{l}\mathrm{OCS} v_{2} P(12-22),{ }^{12} \mathrm{CO} v=1-0 P(21,22), \\
{ }^{13} \mathrm{CO} P(11,12), \mathrm{C}^{18} \mathrm{O} P(10,11), \mathrm{C}^{17} \mathrm{O} P(16)\end{array}$ \\
\hline 2007 Oct 28 & Gemini & 730 & $\mathrm{C}_{2} \mathrm{H}_{2} v_{5} Q$-branch \\
\hline 2007 Oct 30 & Gemini & 2085 & $\begin{array}{c}{ }^{12} \mathrm{CO} v=1-0 P(14-15),{ }^{13} \mathrm{CO} P(3,4), \\
\mathrm{C}^{18} \mathrm{O} P(2,3), \mathrm{C}^{17} \mathrm{O} P(8,9)\end{array}$ \\
\hline
\end{tabular}

most flux of available object types. Toward $5 \mu \mathrm{m}$, most asteroids provide insufficient flux to achieve a high signal-to-noise ratio $(\mathrm{S} / \mathrm{N})$, so we used bright, hot MS stars such as Vega ( $\alpha$ Lyr), Fomalhaut ( $\alpha$ PsA), and Mirfak ( $\alpha$ Per) as divisors. We aimed to observe the divisor for a given target at nearly the same airmass as the target observation for better divisions. Differences in airmass at the times of observations may be corrected after data reduction by taking the divisor spectrum to the power of the ratio of the airmasses. Residual structure in divided spectra was removed by fitting and subtracting low-order polynomials.

Finally, given the high spatial resolution of the Gemini observations, we considered the possibility that TEXES might resolve the emitting/absorbing region around IRS 9 if it were spatially extended. We obtained a series of spatial-spectral scan maps of IRS 9 that allow us to place an upper limit on the linear size of any resolved structure. The approach and results are discussed in detail in Section 5.5.

\subsection{Data Reduction}

Our data reduction pipeline is described in detail in Lacy et al. (2002). Flux calibration is based on the approach of calibrating millimeter and submillimeter data in the manner of Ulich \& Haas (1976). A typical sky calibration cycle involves collecting data on the sky and object with two positions of a rotating chopper blade mounted just above the entrance window to the TEXES Dewar: one painted flat black and one low-emissivity, polished aluminum ("shiny") surface. The result of dividing by the black-sky difference removes atmospheric absorption and serves as both a spatial and spectral flat-field image provided that the black position, sky, and telescope temperatures are approximately equal. We quote the Lacy et al. (2002) expression for the object intensity as a function of frequency,

$$
I_{\nu}(\text { object }) \approx S_{v}(\text { object }- \text { sky }) \frac{B_{v}\left(T_{\text {tel }}\right)}{S_{v}(\text { black }- \text { sky })},
$$

where $S_{v}$ is the measured signal and $B_{v}$ is the blackbody function at the telescope temperature $T_{\text {tel }}$. In the approximation that $I_{v}($ black $)-I_{v}($ sky $)=B_{v}\left(T_{\text {tel }}\right) e^{-\tau_{\text {sky }}}$, this procedure corrects for sky absorption. Wavelength calibration is achieved by using the known wavelengths of night sky emission lines; expressed in velocity units, this method is accurate to $\sim 1 \mathrm{~km} \mathrm{~s}^{-1}$.

Pipeline software written in FORTRAN performs the reduction steps resulting in a wavelength-calibrated and fluxcalibrated object spectrum along with an estimate of the noise, the sky transmission spectrum, and the two-dimensional unextracted slit image. The program also corrects for optical distortion, removes cosmic-ray spikes, and fixes bad pixels. The systematic accuracy of radiometric calibrations is $\sim 25 \%$, ignoring telescope pointing uncertainties.

\section{DESCRIPTION OF THE DATA}

We observed IRS 9 in the lines of seven molecules at the settings summarized in Table 1 . The selection of settings was motivated by the desire to include as many species and individual lines as possible given the severity of telluric atmospheric absorption at a particular setting. In particular, variations in the $\mathrm{H}_{2} \mathrm{O}$ column over Mauna Kea often influenced the settings observed on a particular night, as many of the settings are sensitive to the intensities of telluric water lines. The systemic velocity of IRS 9 and motion of the Earth with respect to the local standard of rest (LSR) were taken into account in choosing settings in which certain lines were shifted either into or away from nearby telluric absorption features, and in many cases required trade-offs in order to maximize efficiency and scientific return. Details of the observations for specific molecules follow.

$$
\text { 3.1. } \mathrm{C}_{2} \mathrm{H}_{2}
$$

At Gemini, two lines in the acetylene $v_{5}$ symmetric bending mode $R$ branch were observed $(J=5,6)$ along with approximately ten lines in the $\nu_{5} Q$ branch. The $R$-branch lines are shown in Figure 1, while Figure 2 shows the $Q$-branch spectrum along with a simple local thermodynamic equilibrium (LTE) fit at a single rotational temperature of $100 \mathrm{~K}$. This is discussed further in Section 4. Each acetylene line appears to consist of a single component. The $R(5)$ line is stronger than $R(6)$, as is expected due to its three-times-greater nuclear statistical weight. $R(6)$ appears to have a weak, redshifted emission component at an LSR velocity of $-54 \mathrm{~km} \mathrm{~s}^{-1}$ although this effect may be 


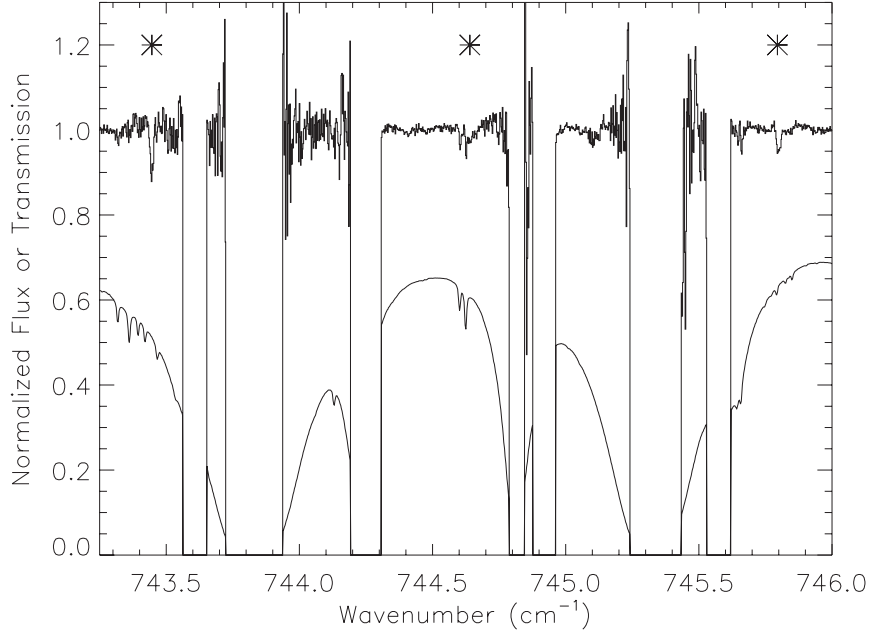

Figure 1. TEXES spectrum toward NGC 7538 IRS 9 showing features due to $\mathrm{C}_{2} \mathrm{H}_{2}$ and $\mathrm{HCN}$. From left to right, the asterisks mark the positions of $\mathrm{C}_{2} \mathrm{H}_{2}$ $v_{5} R(5), \mathrm{HCN} v_{2} R(10)$, and $\mathrm{C}_{2} \mathrm{H}_{2} v_{5} R(6)$. The upper trace shows the data, while the lower curve indicates the relative atmospheric transmission on the same scale. The $\mathrm{HCN} v_{2} R(10)$ line is a detection despite the telluric lines at that wavenumber not completely dividing out. Gaps in the spectrum occur between echelon grating orders and where the telluric absorption is too great to be divided out.

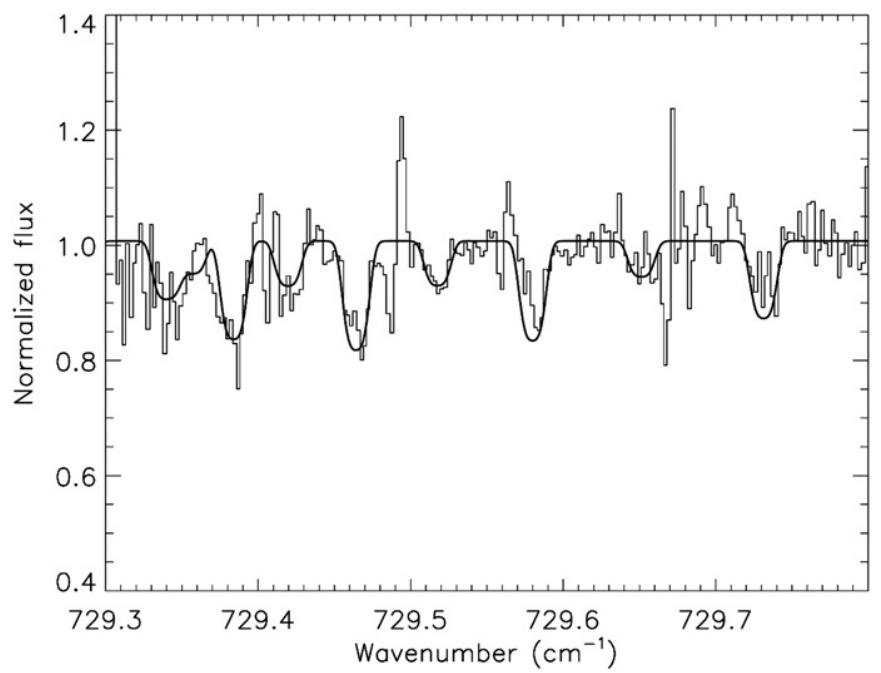

Figure 2. Spectrum of the $v_{5} Q$ branch of $\mathrm{C}_{2} \mathrm{H}_{2}$ observed with TEXES toward NGC 7538 IRS 9 (thin lines). A fit is superimposed from an LTE pure absorption model with $T=100 \mathrm{~K}, b=1 \mathrm{~km} \mathrm{~s}^{-1}$, and $N\left(\mathrm{C}_{2} \mathrm{H}_{2}\right)=2 \times 10^{15} \mathrm{~cm}^{-2}$ (thick line). The synthetic spectrum has been convolved with the TEXES instrumental line shape function.

due to a poor fit to the continuum there. This component can be more easily seen in the top center panel of Figure 10. Earlier IRTF observations of the $\nu_{5} R(1)$ line (wavenumber center $734 \mathrm{~cm}^{-1}$ ) in 2001 June revealed a positive detection, but more importantly, higher $J$ settings $R(15,16)\left(768 \mathrm{~cm}^{-1}\right)$ and $R(21,22)$ $\left(780 \mathrm{~cm}^{-1}\right)$ were non-detections. The latter setting included the ${ }^{2} P_{1 / 2} \rightarrow{ }^{2} P_{3 / 2}$ fine structure line of [Ne II]; consistent with the weak free-free emission observed toward IRS 9, this line was also a non-detection and is discussed in detail in Section 4.4. Finally, although the positions of lines of the ${ }^{13} \mathrm{C}^{12} \mathrm{CH}_{2}$ isotopologue fell in some of our spectral settings, we did not detect any.

We also did not detect any lines in the $2 v_{5}-v_{5}$ "hot bands" of acetylene toward IRS 9. However, the hot-band lines in the spectral settings observed were not particularly favorable for detection, so we cannot draw any strong conclusions about

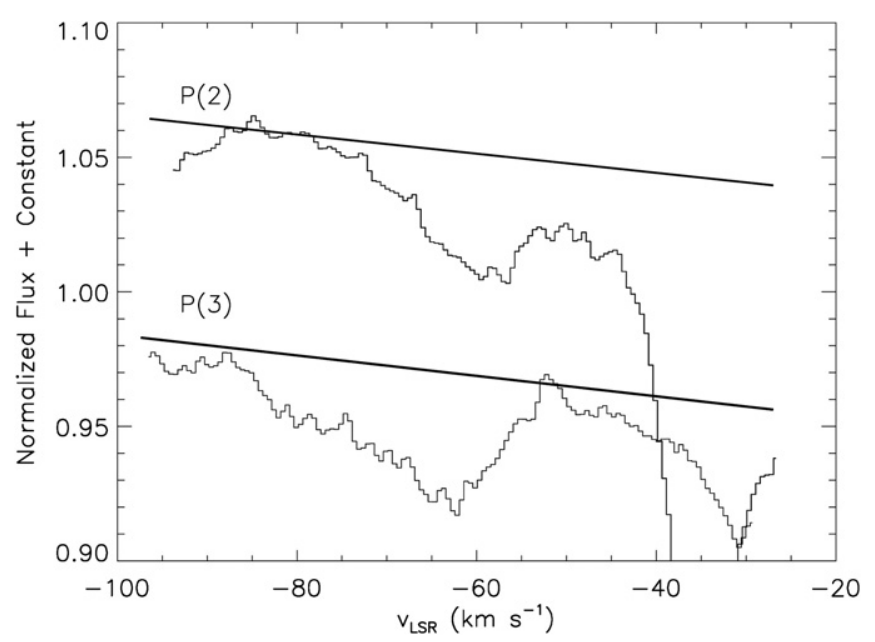

Figure 3. Spectra of two $P$-branch lines of the $v_{4}+v_{5}$ combination mode of $\mathrm{C}_{2} \mathrm{H}_{2}$ shown on an LSR velocity scale. The two spectra have been offset vertically for clarity, and the continua are indicated with heavy straight lines. Both spectra are affected by strong, poorly corrected telluric absorption at small blueshifts.

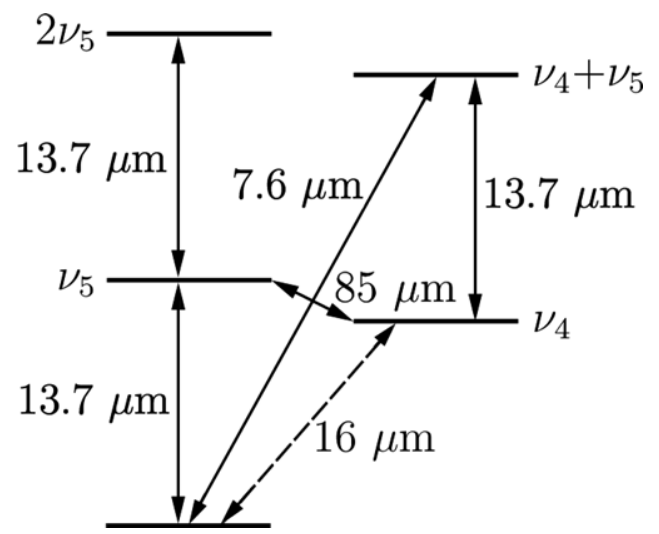

Figure 4. Energy level diagram for some mid-infrared-active, ro-vibrational transitions of $\mathrm{C}_{2} \mathrm{H}_{2}$. Radiatively allowed transitions are denoted with solid lines and collisionally allowed-only transitions with dashed lines. The excited vibrational levels are split by interactions between the rotational angular momentum and the angular momentum of the bending-mode vibration; however, the energy differences between these levels are too small to be seen at the resolution of the diagram.

the $v_{5}$ population from these observations. The observation of $v_{4}+v_{5}$ combination mode lines, shown in Figure 3, is of particular interest. This is because it allows a measurement of the $\mathrm{C}_{2} \mathrm{H}_{2}$ abundance with intrinsically weaker lines at different wavelengths from the $v_{5}$ band lines. Since the $v_{4}+v_{5}$ line strengths are $\sim 5$ times weaker than the $\nu_{5}$ line strengths, absorption in the $v_{4}+v_{5}$ lines may be dominated by high column density clumps of gas.

An energy level diagram indicating relevant vibrational levels of $\mathrm{C}_{2} \mathrm{H}_{2}$ is shown in Figure 4 . The $v_{5}$ mode is the symmetric bending mode in which the $\mathrm{H}$ atoms move together. In the $v_{4}$ antisymmetric bending mode, they move in opposition, resulting in no dipole moment. Therefore, transitions that change the $\nu_{5}$ quantum number are relatively strong, with $A_{u l} \sim 10 \mathrm{~s}^{-1}$. These include the $v_{5}, 2 v_{5}-v_{5}$ (between $2 v_{5}$ and $v_{5}$ ), and $v_{4}+v_{5}-v_{4}$ (between $v_{4}+v_{5}$ and $v_{4}$ ) transitions. Transitions that only change $v_{4}$ do not occur radiatively, but transitions changing both $v_{4}$ and $v_{5}$, including $v_{4}+v_{5}$ and $v_{5}-v_{4}$, do occur although they are $\sim 10$ times weaker than those which only change $\nu_{5}$. Because of these selection rules, $7.6 \mu \mathrm{m}$ photons can be absorbed in $v_{4}+v_{5}$ lines, but molecules excited into the $v_{4}+v_{5}$ level most often decay 
to the $v_{4}$ level from which they can only decay to the ground vibrational level collisionally.

The ratio of the $v_{4}+v_{5} P(3)$ and $P(2)$ lines is difficult to determine due to poor telluric division, but appears to be less than the 9:2 ratio expected from their rotational and nuclear statistical weights. This probably indicates saturation of the lines in spite of their intrinsic weakness relative to the $v_{5}$ transitions. We also note that the spectral region containing the $v_{4}+v_{5}$ lines is contaminated by dense forests of $\mathrm{HNO}_{3}$ and $\mathrm{SO}_{2}$ lines, giving the superficial appearance of bad flat fielding. Another difference between the $v_{5}$ and the $v_{4}+v_{5}$ transitions involves the shapes of the lines; the $v_{4}+v_{5}$ lines are more asymmetric, with blue wings extending to nearly $-90 \mathrm{~km} \mathrm{~s}^{-1}$. In this regard, they resemble the $\mathrm{P}$ Cygni-like shapes of the $\mathrm{CO}$ lines we observed toward IRS 9 (see Section 3.5). The $v_{4}+v_{5}$ lines may result from absorption in a high column density component with a small covering factor that is strongly saturated. Or they may be formed in highly dust-obscured gas that is more visible at 7.6 than $13 \mu \mathrm{m}$ due to the lower dust opacity off of the silicate absorption feature. The presence of the similar $-80 \mathrm{~km} \mathrm{~s}^{-1}$ absorption feature in the $\mathrm{CO}$ lines supports this possibility. Alternately, the $v_{4}+v_{5}$ line strengths relative to the $v_{5}$ lines may be affected by other radiative transfer effects, as we discuss in Section 5.3.

\section{2. $\mathrm{HCN}$}

Three of our spectral settings contain $R$-branch lines of the $v_{2}$ bending mode of $\mathrm{HCN}$ with centers at $734 \mathrm{~cm}^{-1}$ $(6 \leqslant J \leqslant 7), 745 \mathrm{~cm}^{-1}(J=10)$, and $761 \mathrm{~cm}^{-1}(J=16)$. The $R(6)$ line fell into a region where atmospheric transmission is not favorable, and $R(7)$ appears to be a marginal detection because of a weaker, nearby atmospheric feature. $R(10)$ (Figure 1 ) is a probable detection. $R(16)$ falls close to the $R(13)$ line of $\mathrm{C}_{2} \mathrm{H}_{2}$ but is sufficiently separated from the acetylene line to prevent blending and is the only clean $\mathrm{HCN}$ line detected. The $R(15)$ line of $\mathrm{HCN}$ fell just outside the first order of the $761 \mathrm{~cm}^{-1}$ setting. The $\mathrm{S} / \mathrm{N}$ of the $\mathrm{HCN}$ observations is generally lower than that of $\mathrm{C}_{2} \mathrm{H}_{2} \quad v_{5} R$ branch, but the $\mathrm{HCN}$ lines are clearly the broader of the two. The mean full width at half-maximum (FWHM) of the four $\mathrm{C}_{2} \mathrm{H}_{2} R$-branch lines is $6.5 \pm 1 \mathrm{~km} \mathrm{~s}^{-1}$, whereas the HCN $R(16)$ line FWHM is $\sim 15 \mathrm{~km} \mathrm{~s}^{-1}$. Finally, lines of the $\mathrm{H}^{13} \mathrm{CN}$ isotopologue are also found in these settings but not detected: $R(9)$ in $734 \mathrm{~cm}^{-1}$ and $R(18)$ in $761 \mathrm{~cm}^{-1} \cdot R(18)$ falls into a gap between orders and $R(9)$ was affected by poor atmospheric transmission.

\section{3. $\mathrm{CH}_{4}$}

We obtained data on three $v_{4} R$-branch lines of $\mathrm{CH}_{4}$ : the $R(0)$ line at $1311.43 \mathrm{~cm}^{-1}$ and the two $R(2)$ lines at $1322.083 \mathrm{~cm}^{-1}$ and $1322.152 \mathrm{~cm}^{-1}$. In addition, the settings for these lines contained the positions of the $R(1)$ and $R(4)$ lines of ${ }^{13} \mathrm{CH}_{4}$, but both of these lines were clear non-detections. The ${ }^{12} \mathrm{CH}_{4}$ lines show clear asymmetries indicative of multiple velocity components. Each feature has a principal component corresponding to greatest absorption at $v_{\mathrm{LSR}}=-65.0 \mathrm{~km} \mathrm{~s}^{-1}$ with additional components on the red wing of this feature separated in velocity by up to $6.5 \mathrm{~km} \mathrm{~s}^{-1}$. The spectra are plotted in Figure 5 .

\section{4. $\mathrm{NH}_{3}$}

Two spectral settings observed contain a total of ten $P$-branch lines of ammonia, three in the $v_{2}$ "umbrella" mode antisymmetric $P(4, K)$ group and seven in the $v_{2}$ symmetric $P(7, K)$

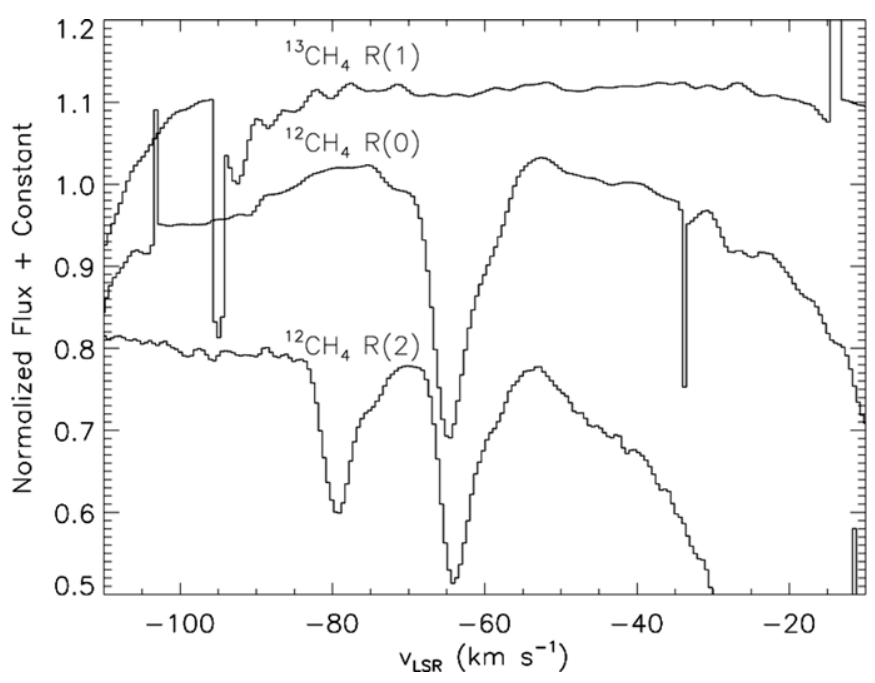

Figure 5. Spectra of three features in the $v_{4}$ band of $\mathrm{CH}_{4}$ toward IRS 9, shown on an LSR velocity scale. For the spectrum containing the $R(2)$ lines, the velocity scale has been set to the LSR value for the stronger component (rest frequency $1322.085 \mathrm{~cm}^{-1}$ ). The individual spectra have been offset vertically for clarity.

group. Of the possible lines in these settings, only $a P(4,2)$ was not observed because of its proximity to a strong telluric atmospheric feature. Almost all of the $\mathrm{NH}_{3}$ lines share a characteristic P Cygni-like profile, with an emission peak near the systemic velocity, around $-60 \mathrm{~km} \mathrm{~s}^{-1}$, and weak, blueshifted absorption separated in velocity by $2.5-10 \mathrm{~km} \mathrm{~s}^{-1}$. This may be compared to the -56.5 and $-59.8 \mathrm{~km} \mathrm{~s}^{-1}$ absorption components seen in mid-infrared $\mathrm{NH}_{3}$ absorption transitions toward IRS 1 (Knez et al. 2009) and a value of $-60.0 \mathrm{~km} \mathrm{~s}^{-1}$ for the $(3,3)$ rotational transition of $\mathrm{NH}_{3}$ in Very Large Array (VLA) observations of IRS 1 at centimeter wavelengths (Henkel et al. 1984). The degree of absorption toward IRS 9 relative to emission varies as well; the highest $K$ lines in the $s P(7, K)$ series show no absorption at all despite the fair $\mathrm{S} / \mathrm{N}$ of the data. Finally, we observed a setting near $930 \mathrm{~cm}^{-1}$ containing the positions of the $\nu_{2}$ $a Q$-branch lines $(1 \leqslant J \leqslant 9)$; the data show weak absorption features at some of the indicated points in Figure 6. For comparison, a similar plot of the $s P(7, K)$ branch spectrum is also shown in the figure. We did not observe lines in the $v_{2} R$-branch of $\mathrm{NH}_{3}$ because of their proximity to regions of significant telluric atmospheric absorption. Also, the $R$-branch lines are located in a spectral region of rapidly increasing absorption due to the $9.7 \mu \mathrm{m}$ silicate dust feature. This fact significantly impacts our interpretation of the $\mathrm{NH}_{3} P$ - and $R$-branch lines, as we discuss in Section 5.3.

\section{5. $\mathrm{CO}$}

We observed two settings in the $5 \mu \mathrm{m} v=1-0$ band of $\mathrm{CO}$, which included a number of $P$-branch lines of several isotopologues. These settings were centered approximately at $2055 \mathrm{~cm}^{-1}$ and $2085 \mathrm{~cm}^{-1}$. Strongest among the detected features were the ${ }^{12} \mathrm{CO}$ lines with $J=14,15,21$, and 22. Each line exhibited a strong $\mathrm{P}$ Cygni profile with emission and absorption components separated by $\sim 20 \mathrm{~km} \mathrm{~s}^{-1}$. Each component was broader than those of any line of any other molecular species we observed, with line widths of $10-20 \mathrm{~km} \mathrm{~s}^{-1}$. In each case the strength of the emission was approximately equal to the strength of the absorption. The features are consistent with an expansion or outflow and have been previously interpreted as such by Mitchell \& Hasegawa (1991). Observations of IRS 1 were also made at these settings; we find a different profile 

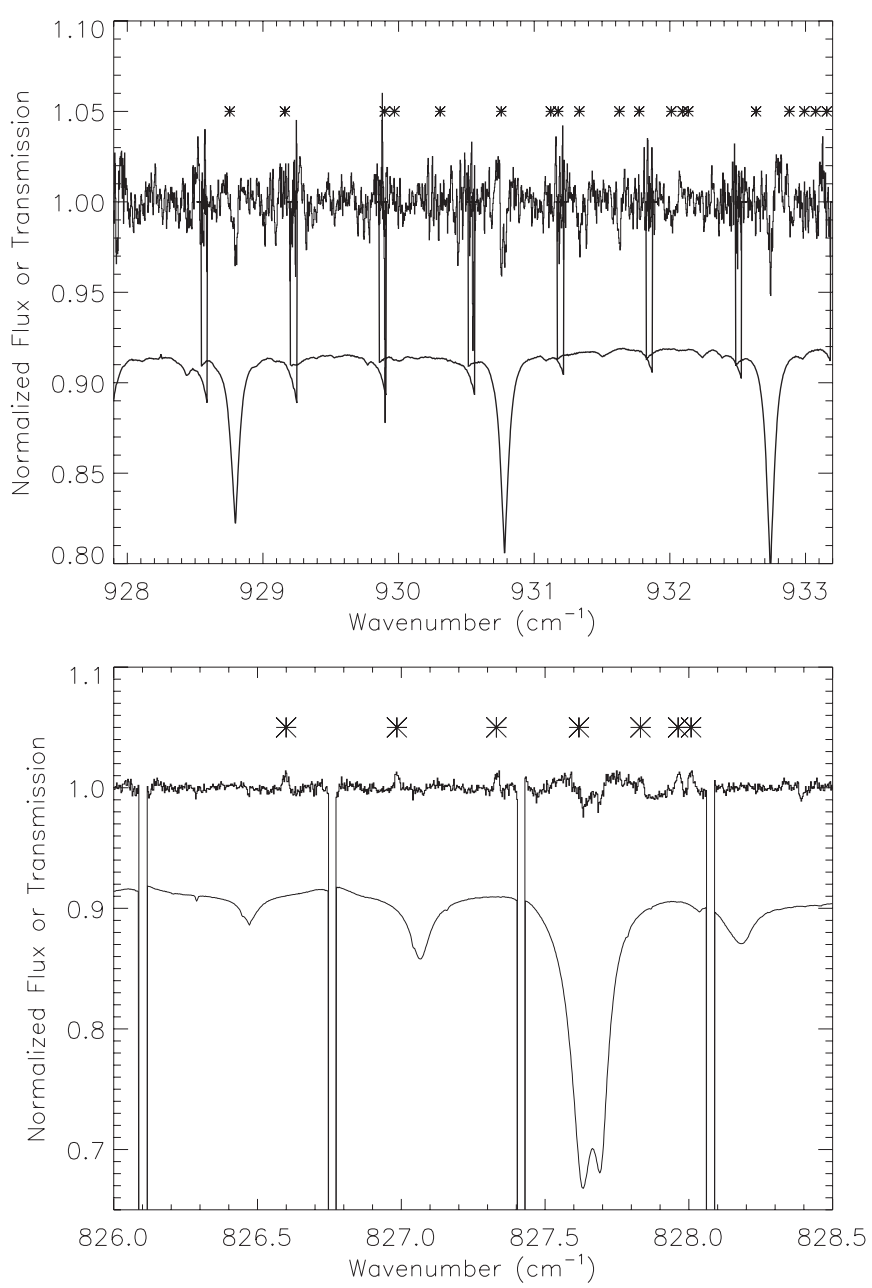

Figure 6. TEXES spectra toward NGC 7538 IRS 9 at the position of the ${ }^{14} \mathrm{NH}_{3}$ $v_{2}$ antisymmetric $Q$ branch (top panel) and symmetric $P(7, K)$ branch (bottom panel). The upper curve in each plot shows the data, while the lower curve in each shows the relative atmospheric transmission on the same scale. Asterisks mark positions of the $\mathrm{NH}_{3}$ lines. Both spectra have been corrected for Earth's motion relative to the LSR, and the position of the $\mathrm{NH}_{3}$ line markers shifted to the systemic LSR velocity of approximately $-60 \mathrm{~km} \mathrm{~s}^{-1}$.

altogether with a double-bottomed absorption feature in ${ }^{12} \mathrm{CO}$, weaker absorption on the blue wing and a slight emission bump on the red wing of each feature. Spectra of both objects are shown in Figure 7.

Four ${ }^{13} \mathrm{CO} P$-branch lines with $J=3,4,11$, and 12 were also detected in the spectrum of IRS 9. Of these, $P(11)$ was a clear detection near the middle of a spectral order and away from contamination by telluric atmospheric lines, $P(12)$ was noisy and found very near the edge of a spectral order, and $P(8)$ and $P(9)$ are clearly detected, but blended slightly with two nearby $\mathrm{C}^{18} \mathrm{O}$ features. Additional ${ }^{13} \mathrm{CO}$ lines, $P(11)$ and $P(12)$, are located in the spectral setting at $2055 \mathrm{~cm}^{-1}$ but occur in regions of strong telluric atmospheric absorption and thus were not detected. We also searched for lines attributable to $\mathrm{C}^{17} \mathrm{O}$ but did not confidently detect any; this is not surprising given the low expected abundance of $\mathrm{C}^{17} \mathrm{O}$. For reference, Smith et al. (2009) quote $N\left({ }^{12} \mathrm{C}^{16} \mathrm{O}\right) / N\left({ }^{12} \mathrm{C}^{17} \mathrm{O}\right)$ of $2800 \pm 300$ toward the $\mathrm{T}$ Tauri star VV CrA, obtained at a spectral resolution similar to our TEXES observations. They compare this to the local ISM value of $2005 \pm 155$ (Wilson 1999).

The CO lines seen toward both IRS 9 and IRS 1 have different line shapes in ${ }^{12} \mathrm{CO}$ and ${ }^{13} \mathrm{CO}$. In the case of IRS 1 ,
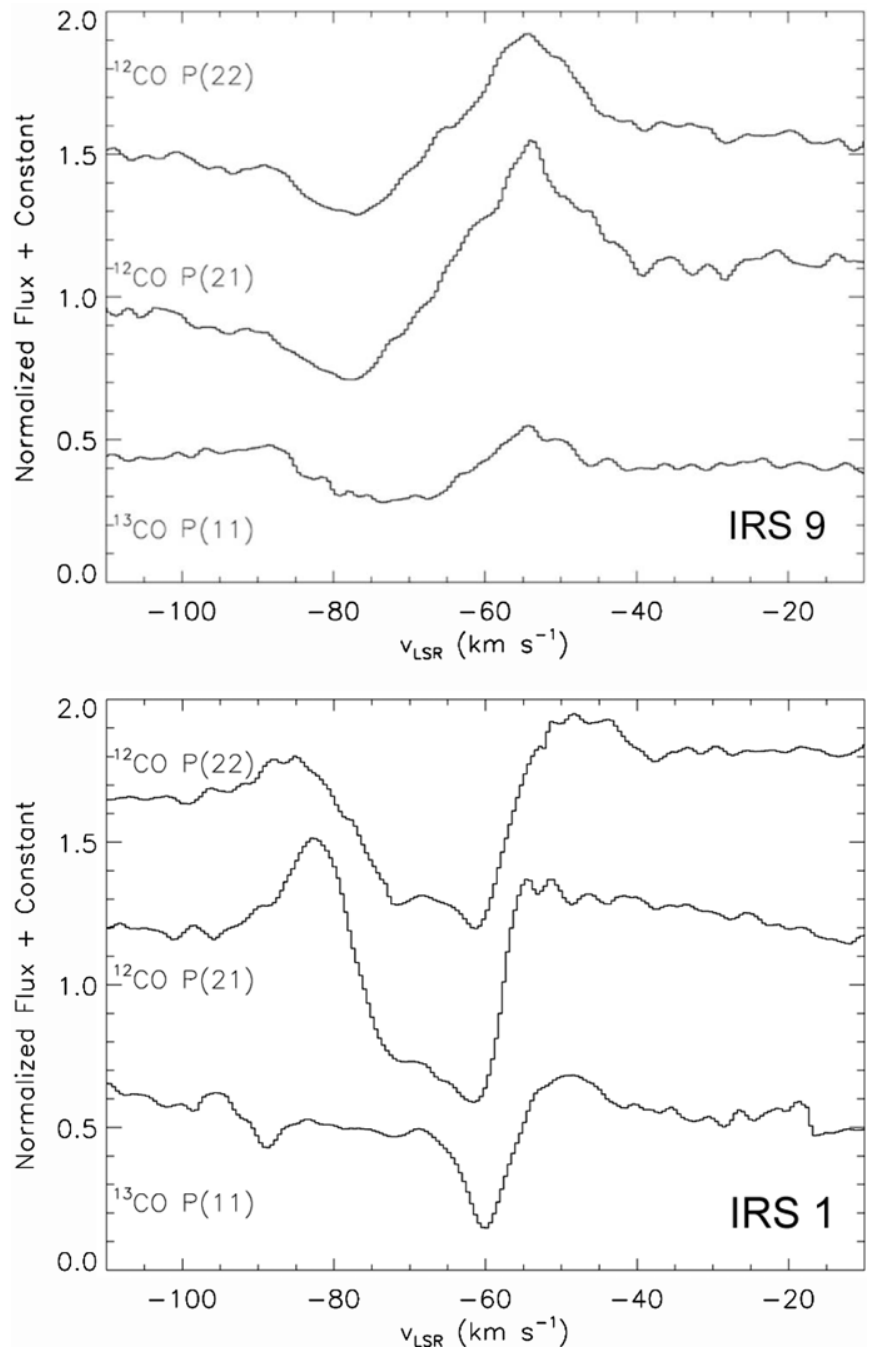

Figure 7. Two $P$-branch lines of ${ }^{12} \mathrm{CO}$ and one $P$-branch line of ${ }^{13} \mathrm{CO}$ in TEXES spectra toward IRS 9 (upper panel) and toward IRS 1 (lower panel). In both cases the motion of the Earth with respect to the LSR has been removed from the velocity scales.

the ${ }^{13} \mathrm{CO}$ features show only single components in the deepest absorption, while toward IRS 9 the ${ }^{13} \mathrm{CO} P(11)$ line and ${ }^{12} \mathrm{CO}$ lines show the pronounced P Cygni profiles. The ${ }^{13} \mathrm{CO}$ lines in the $2085 \mathrm{~cm}^{-1}$ setting clearly show multiple components suggestive of P Cygni profiles in which emission is weak relative to absorption, and there are at least two distinct absorption units. In turn, the strongest absorption components show a weak, double-bottomed structure like the ${ }^{12} \mathrm{CO}$ lines in IRS 1 . The multiple absorption units may appear in the profiles of the ${ }^{12} \mathrm{CO}$ features in the same spectral setting; weak "shoulders" on the blue wing of the absorption component are consistent with additional absorption suffering contamination by the primary components. Such shoulders are also noticeable on the red wings of the emission features of ${ }^{12} \mathrm{CO}$. These details can be seen in Figure 8.

The detection of ${ }^{13} \mathrm{CO}$ toward IRS 9 with line depths comparable to those of ${ }^{12} \mathrm{CO}$ strongly indicates saturation of both isotopologues. Useful columns may however be obtained from rarer variants such as $\mathrm{C}^{18} \mathrm{O}$, but these lines are too weak to be measured accurately in the present spectra. It would also be of interest measure the ${ }^{12} \mathrm{C} /{ }^{13} \mathrm{C}$ ratio toward IRS 9 for comparison with the value of 45 toward IRS 1 reported by Knez et al. (2009). As we did not observe the ${ }^{13} \mathrm{C}$ isotopologue of $\mathrm{C}_{2} \mathrm{H}_{2}$ in 


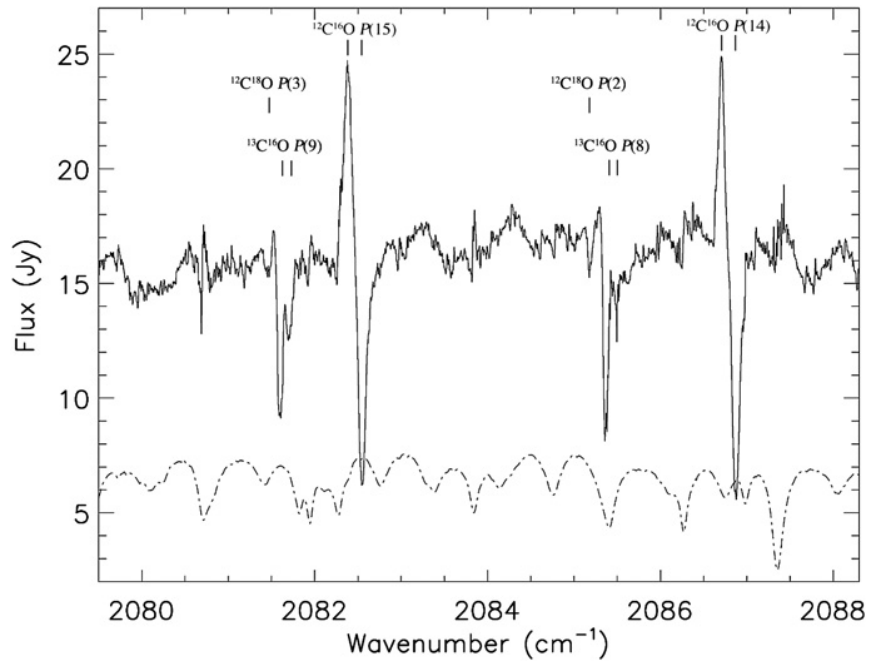

Figure 8. TEXES spectrum toward NGC 7538 IRS 9 centered near the $5 \mu \mathrm{m}$ rotational lines of several isotopologues of CO. The data are shown in the upper trace (solid line) and the relative atmospheric transmission in the lower trace (dot-dashed line) multiplied by a factor of 10 . Some line identifications are shown.

our spectra of IRS 9, the CO observations would offer a way to measure the ratio if the lines were not saturated.

\subsection{OCS}

The ${ }^{12} \mathrm{CO}$ setting at $2055 \mathrm{~cm}^{-1}$ we observed also contains the positions of a number of $P$-branch lines of the $v_{2}$ stretching mode of OCS ( $12 \leqslant J \leqslant 22$ ), but we cannot confidently claim detections of any of them toward IRS 9. Periodic structure in the continuum of our $5 \mu \mathrm{m}$ spectra may be broad OCS features, although it is more likely the structure results from flat-fielding residuals. For reference, we have overplotted the locations of the rest-frame OCS line centers over the data in Figure 9. It is unclear whether we should have observed lines of OCS in the gas phase, given the strong detections of $\mathrm{CO}$ nearby. Low temperatures may inhibit desorption of OCS from grains, but the theoretical grounds for predicting OCS abundances are questionable (Doty et al. 2004). The signature of an outflow in the spectra of CO hints at possible excitation through shocks. We might expect that OCS would be similarly excited but there is no support for this in our data. This is contrasted with the results of Evans et al. (1991), who detected gas-phase OCS toward Orion IRc2; for reasons given in Section 5, it is impossible to know whether this is due to temperature differences or real abundance variations between the IRS 9 and IRS 1 .

\section{7. $\mathrm{HNCO}$}

We looked for $v_{4}$ bending mode HNCO IL-0 $P$-branch lines toward IRS 9 in the TEXES spectral setting that included $s(7, K)$ lines in the $P$ branch of ammonia. None of the three lines included in this setting $(2 \leqslant J \leqslant 4)$ was detected, although there may be HNCO $Q$-branch absorption at $827.7 \mathrm{~cm}^{-1}$, obscured by telluric interference. Given the strong observed ice absorption at $4.62 \mu \mathrm{m}$ toward IRS 9 (Pendleton et al. 1999), now commonly attributed to $\mathrm{OCN}^{-}$, and a lack of gas-phase $\mathrm{HNCO}$ absorption, it seems most likely that temperatures in the outer envelope of IRS 9 are sufficiently low to prevent $\mathrm{OCN}^{-}$desorbing from grains in detectable quantities. Alternately, gas-phase $\mathrm{OCN}^{-}$ might exist at $\mathrm{H}$ (or $\mathrm{H}_{2}$ ) abundances insufficient to result in considerable $\mathrm{HNCO}$ production.
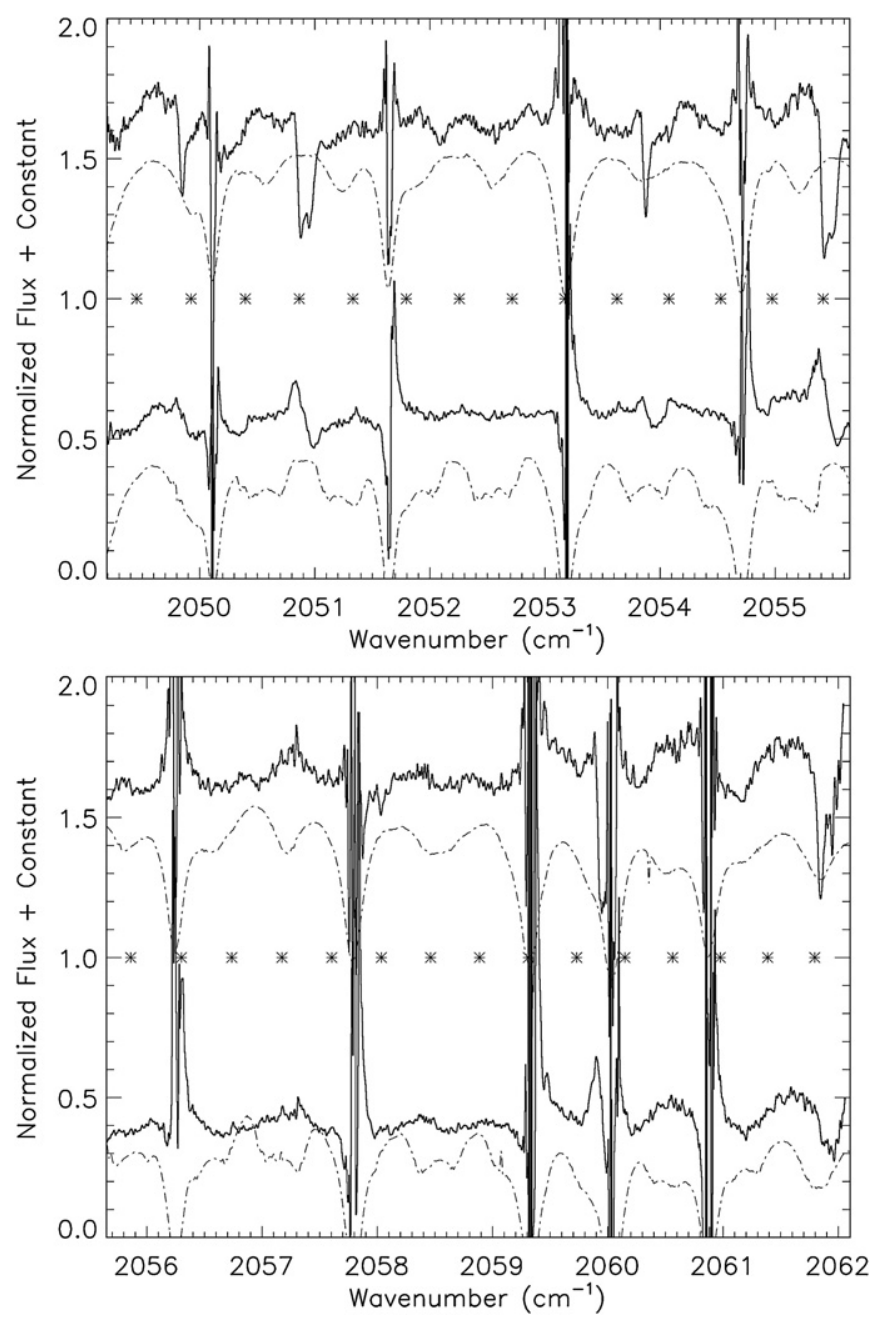

Figure 9. TEXES spectra of NGC 7538 IRS 1 (top) and IRS 9 (bottom) near features in the $P$ branches of ${ }^{12} \mathrm{CO}$ and ${ }^{13} \mathrm{CO}$; heavy solid lines are the object spectra and light dot-dash lines are the sky spectra offset for clarity. The wavenumber scale has been corrected to a velocity reference at rest in each object's frame, and the line centers of OCS features, marked with asterisks, have been velocity shifted appropriately.

\section{ANALYSIS AND MODELING}

We fit synthetic spectra to the TEXES data to derive excitation parameters and column densities for the various molecular species observed. A two-step process was devised in which rough estimates of the parameters were made from "by eye" fits of synthetic spectra to our data; these values were then used as the initial guesses in our fitting program which iterates values of hundreds parameters to achieve the best fit to the data by minimizing $\chi^{2}$. While our $\chi^{2}$ code is robust in finding solutions even when starting far from the global minimum, convergence is achieved faster by using good initial guesses. This approach was best suited to our method of spectral analysis.

\subsection{Simple Synthetic Spectrum Fitting}

In order to get approximate parameters for the gas-phase lines we observed, we first fit synthetic spectra to the data using a fitting method commonly referred to as "chi by eye." This approach allowed us to get quick initial estimates of parameters such as excitation temperature and column density for the various molecules that were used as inputs to a more sophisticated nonlinear least-squares code to obtain optimal 
Table 2

Molecular Absorption Parameters Derived from Simple Synthetic Spectrum Fitting in LTE

\begin{tabular}{|c|c|c|c|c|c|c|c|c|c|c|c|c|}
\hline Molecule/Band & Line & $n^{\mathrm{a}}$ & $\begin{array}{c}T \\
(\mathrm{~K})\end{array}$ & $\begin{array}{c}v_{1}^{\mathrm{b}} \\
\left(\mathrm{km} \mathrm{s}^{-1}\right)\end{array}$ & $v_{2}$ & $v_{3}$ & $\begin{array}{c}b_{1} \\
\left(\mathrm{~km} \mathrm{~s}^{-1}\right)\end{array}$ & $b_{2}$ & $b_{3}$ & $\begin{array}{c}N_{1} \mathrm{c} \\
\left(\mathrm{cm}^{-2}\right)\end{array}$ & $N_{2}$ & $N_{3}$ \\
\hline \multirow[t]{4}{*}{$\mathrm{C}_{2} \mathrm{H}_{2} v_{5}$} & $R(1)$ & 1 & 100 & -62.0 & $\ldots$ & $\ldots$ & 3.3 & $\ldots$ & $\ldots$ & $1.0(15)$ & $\ldots$ & $\ldots$ \\
\hline & $R(5)$ & 1 & 100 & -62.0 & $\ldots$ & $\ldots$ & 2.4 & $\ldots$ & $\ldots$ & $4.5(14)$ & $\ldots$ & $\ldots$ \\
\hline & $R(6)$ & 1 & 100 & -65.5 & $\ldots$ & $\ldots$ & 2.4 & $\ldots$ & $\ldots$ & $4.5(14)$ & $\ldots$ & $\ldots$ \\
\hline & $Q$ branch & 1 & 100 & $\ldots$ & $\ldots$ & $\ldots$ & 1.0 & $\ldots$ & $\ldots$ & $2.0(15)$ & $\ldots$ & $\ldots$ \\
\hline $\mathrm{C}_{2} \mathrm{H}_{2} v_{4}+v_{5}$ & $\mathrm{P}(2)$ & 1 & 100 & -61.0 & $\ldots$ & $\ldots$ & 6.8 & $\ldots$ & $\ldots$ & $2.5(16)$ & $\ldots$ & $\ldots$ \\
\hline $\mathrm{HCN} v_{2}$ & $R(16)$ & 1 & 200 & -64.5 & $\ldots$ & $\ldots$ & 7.9 & $\ldots$ & $\ldots$ & $1.2(16)$ & $\ldots$ & $\ldots$ \\
\hline \multirow[t]{3}{*}{${ }^{12} \mathrm{CH}_{4} v_{4}$} & $R(0)^{\mathrm{d}}$ & 4 & 200 & -65.0 & -54.0 & -61.0 & 2.3 & 2.7 & 4.6 & $7.5(16)$ & $-4.0(15)$ & $6.0(16)$ \\
\hline & $R(2)$ & 2 & 200 & -65.5 & -61.5 & $\ldots$ & 2.3 & 2.5 & $\ldots$ & $6.0(16)$ & $3.3(16)$ & $\ldots$ \\
\hline & $R(2)$ & $\ldots$ & $\ldots$ & $\ldots$ & $\ldots$ & $\ldots$ & 2.5 & 2.3 & $\ldots$ & $6.3(16)$ & $1.5(16)$ & $\ldots$ \\
\hline \multirow[t]{9}{*}{${ }^{14} \mathrm{NH}_{3} v_{2}$} & $a P(4,0)$ & 2 & 200 & -64.0 & -70.0 & $\ldots$ & 4.6 & 3.9 & $\ldots$ & $-2.5(15)$ & $5.0(14)$ & $\ldots$ \\
\hline & $a P(4,1)$ & 2 & 200 & -62.0 & -72.0 & $\ldots$ & 5.3 & 3.9 & $\ldots$ & $-3.8(15)$ & $2.5(14)$ & $\ldots$ \\
\hline & $a P(4,3)$ & 2 & 200 & -62.0 & -70.0 & $\ldots$ & 2.8 & 3.9 & $\ldots$ & $-1.0(15)$ & $1.0(15)$ & $\ldots$ \\
\hline & $s P(7,1)$ & 2 & 200 & -62.0 & -70.0 & $\ldots$ & 1.8 & 4.0 & $\ldots$ & $-2.0(15)$ & $7.5(14)$ & $\ldots$ \\
\hline & $s P(7,2)$ & 2 & 200 & -61.0 & -62.5 & $\ldots$ & 3.3 & 2.9 & $\ldots$ & $-6.0(15)$ & $4.5(15)$ & $\ldots$ \\
\hline & $s P(7,3)$ & 2 & 200 & -58.5 & -64.0 & $\ldots$ & 1.8 & 2.9 & $\ldots$ & $-1.2(15)$ & $1.5(15)$ & $\ldots$ \\
\hline & $s P(7,4)$ & 2 & 200 & -60.5 & -63.0 & $\ldots$ & 1.8 & 1.8 & $\ldots$ & $-2.5(15)$ & $2.0(15)$ & $\ldots$ \\
\hline & $s P(7,5)$ & 1 & 200 & -56.0 & $\ldots$ & $\ldots$ & 4.4 & $\ldots$ & $\ldots$ & $-7.0(15)$ & $\ldots$ & $\ldots$ \\
\hline & $s P(7,6)$ & 1 & 200 & -57.0 & $\ldots$ & $\ldots$ & 4.4 & $\ldots$ & $\ldots$ & $-4.0(15)$ & $\ldots$ & $\ldots$ \\
\hline \multirow[t]{4}{*}{${ }^{12} \mathrm{C}^{16} \mathrm{O} v=1-0$} & $P(14)$ & 3 & 200 & -55.0 & -64.5 & -88.5 & 2.4 & 6.5 & 7.2 & $-1.0(16)$ & $-1.1(17)$ & $3.0(17)$ \\
\hline & $P(15)$ & 3 & 200 & -51.5 & -63.5 & -88.5 & 3.6 & 9.4 & 7.2 & $-2.5(16)$ & $-2.2(17)$ & $3.0(17)$ \\
\hline & $P(21)$ & 2 & 200 & -54.5 & -77.5 & $\ldots$ & 10.9 & 10.9 & $\ldots$ & $-8.0(17)$ & $5.5(17)$ & $\ldots$ \\
\hline & $P(22)$ & 2 & 200 & -55.0 & -77.0 & $\ldots$ & 8.0 & 10.2 & $\ldots$ & $-6.0(17)$ & $6.0(17)$ & $\ldots$ \\
\hline \multirow[t]{4}{*}{${ }^{13} \mathrm{C}^{16} \mathrm{O} v=1-0$} & $P(3)$ & 3 & 200 & -60.5 & -70.5 & -89.0 & 4.3 & 4.3 & 1.4 & $-9.0(17)$ & $8.0(18)$ & $7.0(17)$ \\
\hline & $P(4)$ & 3 & 200 & -61.5 & -71.5 & -86.0 & 3.6 & 5.0 & 6.5 & $-7.0(17)$ & $6.8(18)$ & $3.4(18)$ \\
\hline & $P(11)$ & 2 & 200 & -54.0 & -75.0 & $\ldots$ & 5.8 & 10.2 & $\ldots$ & $-8.0(17)$ & $2.7(18)$ & $\ldots$ \\
\hline & $P(12)$ & 2 & 200 & -54.0 & -73.0 & $\ldots$ & 5.9 & 10.2 & $\ldots$ & $-3.5(17)$ & $6.5(18)$ & $\ldots$ \\
\hline${ }^{12} \mathrm{C}^{18} \mathrm{O} v=1-0$ & $P(2)$ & 2 & 200 & -68.0 & -72.0 & $\ldots$ & 2.9 & 3.6 & $\ldots$ & $7.5(18)$ & $7.5(18)$ & $\ldots$ \\
\hline
\end{tabular}

Notes.

a Number of velocity components fitted.

b All velocities are referred to the LSR.

c Column densities are given in the form $A(B)=A \times 10^{B}$. Negative values of $A$ indicate components in emission. "Column density" includes all rotational states, assuming $T=200 \mathrm{~K}$

d The best fit to this line required four components. The remaining component not in the table is $v_{4}=-71.5 \mathrm{~km} \mathrm{~s}-1, b_{4}=2.3 \mathrm{~km} \mathrm{~s}$, and $N_{4}=$ $5.0(15) \mathrm{cm}^{-2}$.

values of the parameters. The simple method also allowed us to determine the number, width, and velocities of line components.

The equivalent width of an optically thin line is the product of the line strength, $\alpha$, and the column density, $N$. We obtained values of $\alpha$ for each molecular species observed from the GEISA database $^{1}$ of laboratory molecular data (Jacquinet-Husson et al. 2005). The GEISA coefficients are specified for a fiducial temperature $T_{o}=296 \mathrm{~K}$; we corrected them to an assumed temperature $T$ via the formula

$$
\alpha(T)=\alpha\left(T_{o}\right) \exp \left[E_{l}\left(\frac{1}{k_{B} T_{o}}-\frac{1}{k_{B} T}\right)\right]\left(\frac{T_{o}}{T}\right)^{\beta},
$$

where $E_{l}$ is the energy of the lower state of a transition, $k_{B}$ is Boltzmann's constant, and $\beta=1$ for linear molecules and $3 / 2$ for nonlinear molecules. Here, the temperature dependence of the vibrational partition function and the stimulated emission correction are neglected as both are of order unity. For Gaussian lines, the line-center optical depth is calculated according to

$$
\tau_{l}=\frac{N \alpha}{b \sqrt{\pi}}
$$

\footnotetext{
1 http://ether.ipsl.jussieu.fr/ether/pubipsl/geisa_iasi_raie_frame_2003_uk.jsp
}

where $b$ is the Doppler $b$ parameter in wavenumber units. The run of optical depth with frequency over the line is computed with

$$
\tau(w)=\tau_{l} \exp \frac{-\left(w-w_{o}\right)^{2}}{b^{2}}
$$

in which $w$ is the wavenumber and $w_{o}$ is the line-center wavenumber. The optical depths are converted to intensities by taking the exponential of $\tau(w)$; the continua are normalized to unity. We assume that the rotational states are in LTE. Our radiative transfer model also assumes pure absorption for fitting the observed absorption lines. Re-emission by the absorbing molecules is neglected. Emission features are treated as separate components, which are modeled as negative absorption. Populations of excited vibrational states are not calculated. Temperatures are arbitrarily held fixed at $200 \mathrm{~K}$; this value is consistent with temperatures around IRS 1 derived from TEXES data. The exception was the $Q$-branch of $\mathrm{C}_{2} \mathrm{H}_{2}$ where a number of observed $J$ states allowed the temperature to be constrained to $100 \mathrm{~K}$. Column densities and velocities derived using this method are shown in Table 2. Fits to the individual lines are shown in Figures 10 through 12. 

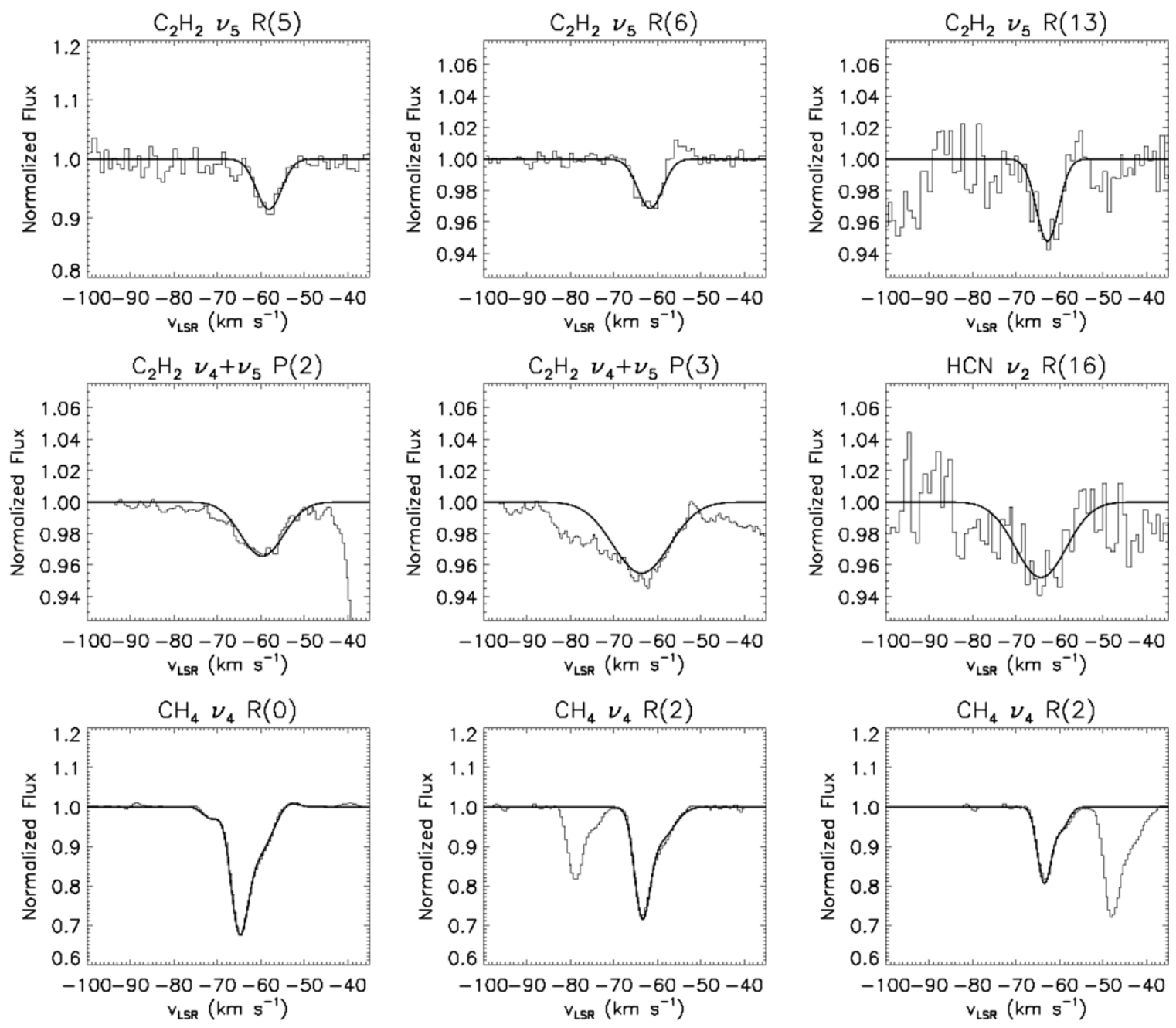

Figure 10. TEXES spectra of NGC 7538 IRS 9 (thin lines) and synthetic spectra computed using the simple LTE fitting method described in Section 4.1 (thick lines). The continuum in each spectrum has been normalized to unity and the spectra are plotted on a velocity scale referred to the LSR. Features shown are $\mathrm{C}_{2} \mathrm{H}_{2} v_{5} R(5)$, $R(6)$, and $R(13) ; \mathrm{C}_{2} \mathrm{H}_{2} v_{4}+v_{5} P(2)$ and $P(3) ; \mathrm{HCN} v_{2} R(16)$; and $\mathrm{CH}_{4} v_{4} R(0)$ and the two components of $R(2)$.

\section{2. $\chi^{2}$ Minimization Spectral Fitting}

Our spectral fitting code, FITSPEC, uses the LevenbergMarquardt method of inverting the second derivative matrix to minimize $\chi^{2}$ as in Bevington \& Robinson (2003) for up to four unique absorbing or emitting units, or "clouds," along the line of sight. The one-dimensional radiative transfer model assumes rotational LTE of all molecules with a single excitation temperature. As in our "by eye" fitting, re-emission by absorbing molecules is neglected and emission features are modeled as negative absorption. The total number of free fitting parameters depends on the number of molecules in the fit and the number of echelon orders in a particular spectral setting. Although there is a large number of free parameters in the fit, most of them are "uninteresting," such as baseline coefficients. The "interesting" parameters in the fit are Doppler shift, Doppler $b$ parameter, column density, temperature, and covering factor for each cloud and for each molecule. The details of the program are discussed in Knez et al. (2009). For our observed molecules, we chose a number of absorbing clouds consistent with the number of velocity components seen in the TEXES spectra, allowing FITSPEC to independently determine their parameters. When available, we included data indicating the non-detection of certain lines, such as high- $J \mathrm{C}_{2} \mathrm{H}_{2}$ near $13 \mu \mathrm{m}$, to better constrain the fit parameters.
The success of FITSPEC requires accurate molecular transition data to compute synthetic spectra against which to fit the data. We relied on the calculations in Evans et al. (1991), giving information including the line center, the energy of the transition either in units of $J(J+1)$ (which is then multiplied by the molecule's rotational constant $B$ ) or $E$ in units of $\mathrm{cm}^{-1}$, and the rotational line strengths (Hönl-London factors) in units of $\mathrm{cm}^{-1}\left(10^{16} \mathrm{~cm}^{-2}\right)^{-1}$. The program uses guesses of the starting parameter values to compute the initial model spectrum and then iterates the fit by varying those values until a convergence criterion is satisfied. The requirement is

$$
\sum_{i}\left(\frac{\Delta p_{i}}{\sigma_{p_{i}}}\right)^{2} \leqslant 0.01
$$

where $\Delta p_{i}$ is the change in the value of parameter $i$ and $\sigma_{p_{i}}$ is the uncertainty in the parameter $i$, allowing all other parameters to vary.

The output of the program consists of the best-fit parameter values, uncertainty estimates, and a synthetic spectrum having the properties of the best fit. The intensity of the synthetic spectrum is calculated by taking $I=I_{o} e^{-\tau}$ of each absorbing unit along the line of sight and adding any optically 

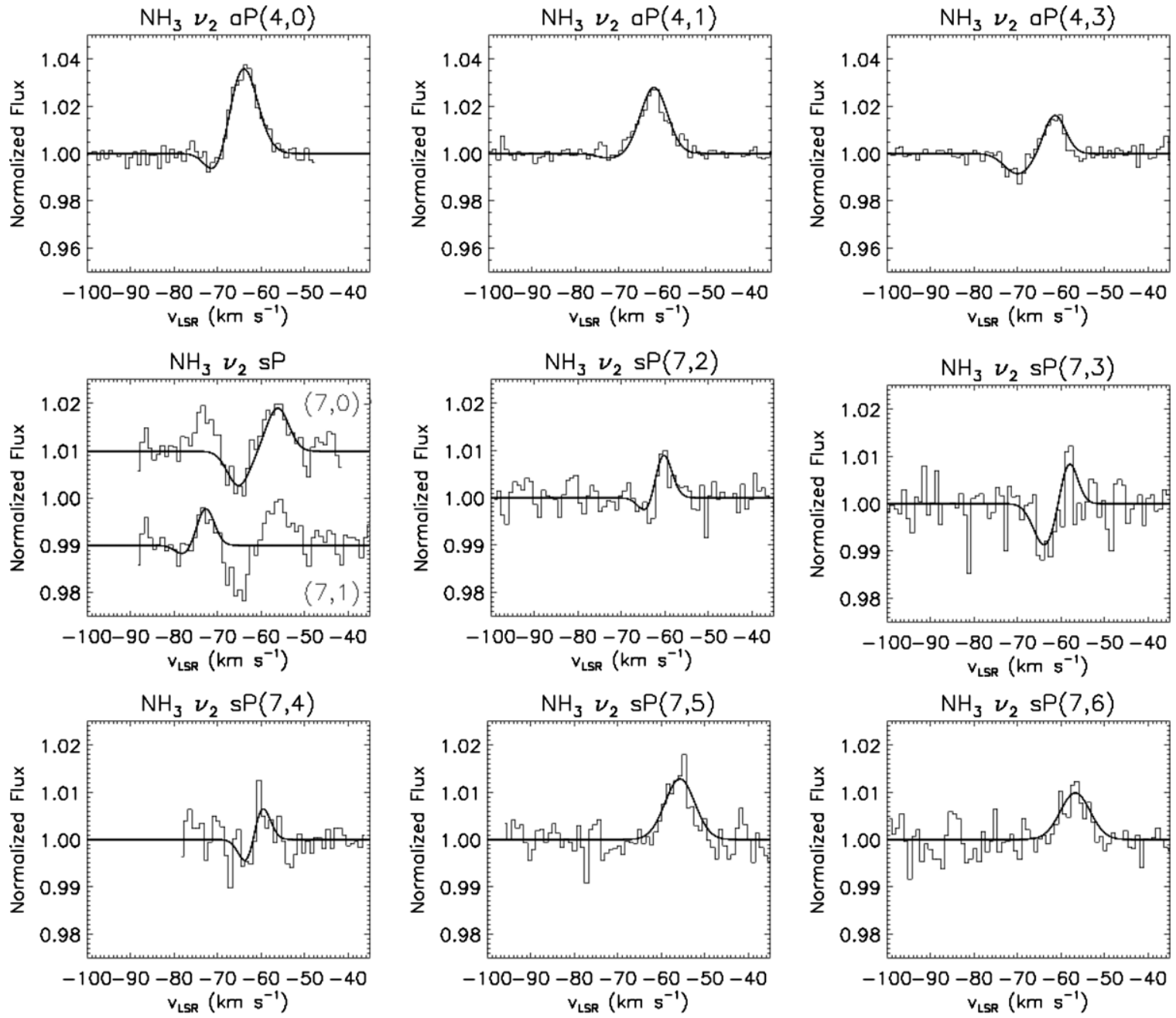

Figure 11. TEXES spectra of NGC 7538 IRS 9 (continued). Features shown are $\mathrm{NH}_{3} v_{2} a P(4,0), a P(4,1), a P(4,3), s P(7,0), s P(7,1), s P(7,2), s P(7,3), s P(7,4), s P(7,5)$, and $s P(7,6)$. The $s P(7,0)$ and $s P(7,1)$ lines are shown together in a single panel using the velocity scale for $s P(7,0)$ and are offset slightly for clarity.

thin emission as

$$
I_{\mathrm{obs}}=I_{0} \prod_{n=1}^{N}\left[1-C_{n}\left(1-e^{-\tau_{n}(v)}\right)\right]+S_{\mathrm{em}} C_{\mathrm{em}} \tau_{\mathrm{em}}(v),
$$

where $N$ is the number of absorbing clouds in the fit, $I_{0}$ is the intensity of radiation from the background continuum source (set to unity since the spectra are normalized), $C_{n}=\Omega_{n} / \Omega_{0}$ and $\tau_{n}$ are the covering factors and optical depths of the absorbing clouds, $S_{\mathrm{em}}$ and $C_{\mathrm{em}}=\Omega_{\mathrm{em}} / \Omega_{0}$ are the source function and covering factor of the emitting material, and $\Omega_{0}$ is the solid angle of the continuum source. The right-hand term represents an optically thin emitting cloud; since it only depends on the product of $S_{\mathrm{em}}$ and $C_{\mathrm{em}}$, we scale $C_{\mathrm{em}}$ by $S_{\mathrm{em}} / I_{0}$ and drop $S_{\mathrm{em}}$ from the formula. The product term in Equation (6) assumes that the absorbing clouds overlap randomly. That is, if two clouds each have covering factors of $0.1,1 / 10$ of each lies along the line of sight to the other.

Knez et al. (2009) used the same approach and note this results in greater absorption in saturated lines than would result if the optical depths were added first and then the transmission spectrum were calculated from the optical depth spectrum. The resulting fits to the data are better than those obtained by summing the optical depths first and then taking the exponential. This fitting approach, however, is not without drawbacks. Emission lines may not be thermally excited, but rather could be due to resonantly scattered light; we have no way of discriminating between these processes. We assume that upward transitions in the molecules we observe are not followed by re-emission of that radiation; in reality, most absorptions should be followed by re-emission, although in a direction uncorrelated with the incoming radiation, so the extent to which re-emission fills in the absorption depends on the distribution of the re-emitting gas around the continuum source.

The results of our FITSPEC runs on the IRS 9 data are summarized in Table 3 where they are compared with results from fitting TEXES data of IRS 1 with the same procedure in Knez et al. (2009).

\subsection{Column Density Upper Limits For Non-detections}

We calculate column density upper limits from the lines that were not detected in the spectrum of IRS 9 by convolving the data with a Gaussian line shape function whose properties are consistent with those of detected lines. We begin by assuming that a normalized spectrum containing a single absorption line has the functional form

$$
F_{v}=1-a e^{-x^{2} / b^{2}},
$$

where $a>0$ is the depth of the line and $b$ is the Doppler $b$ parameter. The observed spectrum is convolved with a Gaussian 

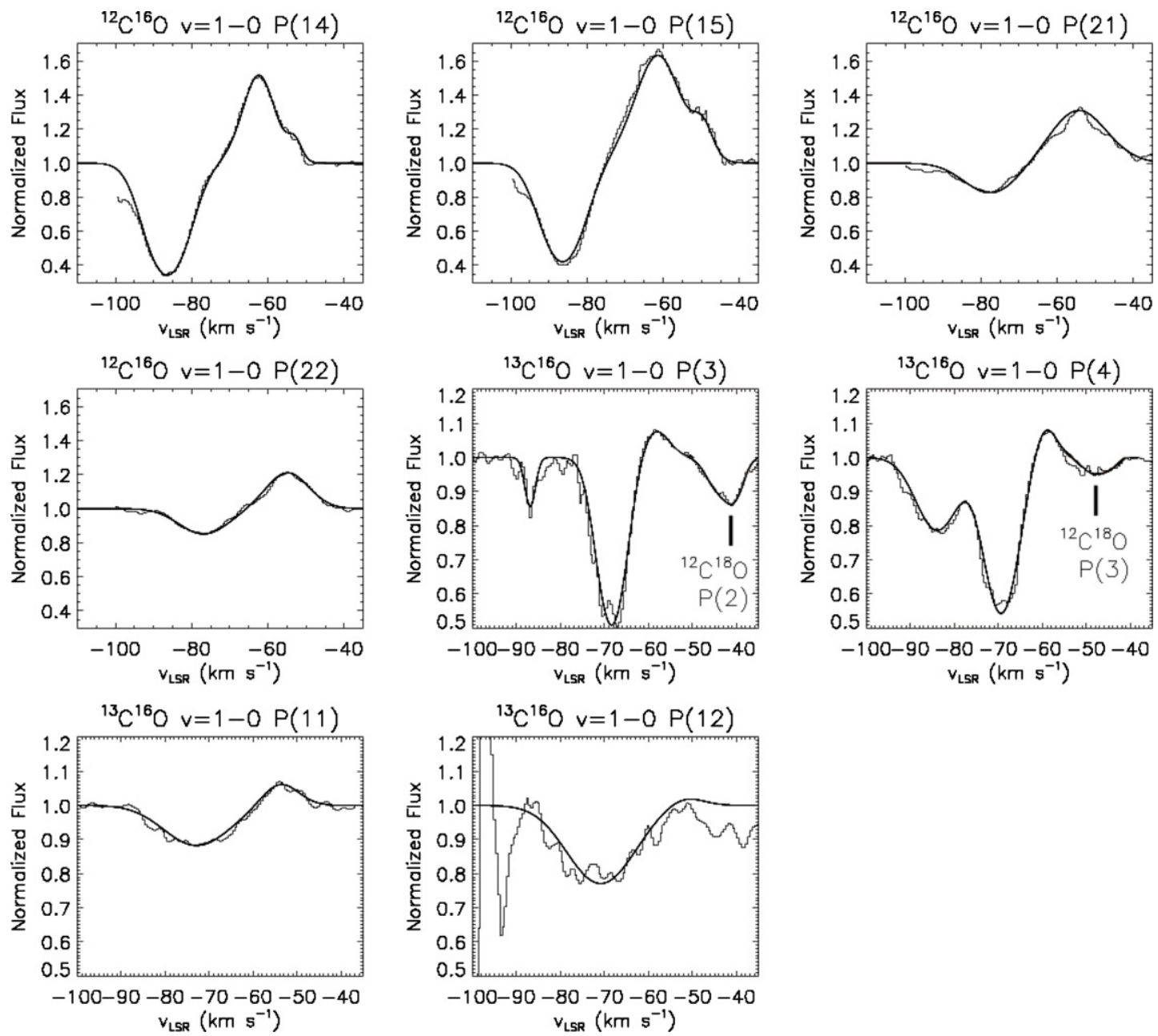

Figure 12. TEXES spectra of NGC $7538 \mathrm{IRS} 9$ (continued). Features shown are ${ }^{12} \mathrm{C}^{16} \mathrm{O} v=1-0 P(14), P(15), P(21)$, and $P(22) ;{ }^{13} \mathrm{C}^{16} \mathrm{O} v=1-0 P(3) /{ }^{12} \mathrm{C}^{18} \mathrm{O} v=$ $1-0 P(2) ;{ }^{13} \mathrm{C}^{16} \mathrm{O} v=1-0 P(4) /{ }^{12} \mathrm{C}^{18} \mathrm{O} v=1-0 P(3)$; and ${ }^{13} \mathrm{C}^{16} \mathrm{O} v=1-0 P(11)$ and $P(12)$.

of the form $n e^{-x^{2} / b^{2}}$ where $n$ is a normalization factor here chosen to be $1 / \sqrt{\pi} b$ such that the integral of the Gaussian is unity. The convolution is given by

$$
F_{\nu} \otimes n e^{-x^{2} / b^{2}}=\int F_{v} n e^{-x^{2} / b^{2}} d \nu,
$$

where $x=v-v^{\prime}$. The upper limit of the equivalent width, $W_{\max }$, of a line not detected is proportional to the peak value of the convolved spectrum according to

$$
W_{\max }=\sqrt{\pi} P b
$$

in which $P$ is the peak. We used $b=3.5 \mathrm{~km} \mathrm{~s}^{-1}$ in making the calculations, a value consistent with those derived by spectral fitting methods described previously. The column density limit for species $X$ was then computed from

$$
N(X)=\frac{W_{\max }}{\alpha},
$$

where $\alpha$ is the line strength. We used the GEISA line strengths where available and corrected them to a uniform temperature of $200 \mathrm{~K}$ as described previously. Results for our non-detections are given in Table 4.

We derived upper limits for all lines of a particular molecular species available in a given spectral setting, as indicated in the table by $n$, the number of lines included in the fit. In cases where $n>1$, the equivalent width and column density upper limits given are simple averages. All limits are given with respect to the total number of molecules along the column, irrespective of isotopic composition except for ${ }^{13} \mathrm{CH}_{4}$; here we have divided the derived column density by the terrestrial ${ }^{12} \mathrm{C} /{ }^{13} \mathrm{C}=89$ ratio built into GEISA to recover the column density specifically for ${ }^{13} \mathrm{CH}_{4}$.

\subsection{An Upper Limit to the Ionizing Flux in IRS 9}

The ${ }^{2} P_{1 / 2} \rightarrow{ }^{2} P_{3 / 2}$ fine structure line of [Ne II] at $780.42 \mathrm{~cm}^{-1}$ is often used as a diagnostic of ionized gas at our wavelengths. The non-detection of this line in the spectrum of IRS 9 places a constraint on the amount of ionizing flux being emitted by the embedded central source.

The Lyman continuum luminosity $\left(N_{\text {Lyc }}\right)$ can be calculated from the [Ne II] luminosity since both are proportional to the square of the density integrated over volume. The [Ne II] volume emissivity is given by

$$
j_{[\mathrm{Ne} \mathrm{II}]}=n_{e} n_{\mathrm{Ne}^{+}} q_{l u} h v \frac{n_{c}}{n_{e}+n_{c}},
$$

where $n_{e}$ is the electron density, $n_{\mathrm{Ne}^{+}}$is the density of ionized neon, $q_{l u}$ is the rate coefficient for collisional excitation $\left(q_{l u} h \nu=8.4 \times 10^{-22} \mathrm{erg} \mathrm{s}^{-1} \mathrm{~cm}^{3}\right)$, and $n_{c}=$ $A_{u l} / q_{u l}\left(=4.9 \times 10^{5} \mathrm{~cm}^{-3}\right.$ at an electron temperature $T_{e}=$ 
Table 3

Molecular Absorption Parameters Determined from $\chi^{2}$ Minimization Spectral Fitting

\begin{tabular}{|c|c|c|c|c|c|}
\hline Species & $\begin{array}{c}v_{\mathrm{LSR}} \\
\left(\mathrm{km} \mathrm{s}^{-1}\right)\end{array}$ & $\begin{array}{l}T_{\mathrm{ex}} \\
(\mathrm{K})\end{array}$ & $\begin{array}{c}b \\
\left(\mathrm{~km} \mathrm{~s}^{-1}\right)\end{array}$ & $\begin{array}{c}N(X)^{\mathrm{a}} \\
\left(10^{16} \mathrm{~cm}^{-2}\right)\end{array}$ & $\begin{array}{c}\mathrm{C} \\
(\%)\end{array}$ \\
\hline \multicolumn{6}{|c|}{ IRS 9 (this work) } \\
\hline \multirow[t]{2}{*}{$\mathrm{C}_{2} \mathrm{H}_{2}$} & $-58.9(1.4)$ & $195(57)$ & $4.0(1.3)$ & $-0.2(0.1)$ & $\overline{52(189)^{b}}$ \\
\hline & $-63.9(1.0)$ & 205(49) & $4.0(1.1)$ & $2.3(0.7)$ & $16(9)$ \\
\hline \multirow[t]{2}{*}{$\mathrm{HCN}$} & $-58.7(1.7)$ & $200(68)$ & $4.0(1.3)$ & $0.5(0.7)$ & $52(193)^{b}$ \\
\hline & $-63.8(2.9)$ & $204(76)$ & $4.0(1.4)$ & $3.9(4.3)$ & 11(6) \\
\hline \multirow[t]{2}{*}{$\mathrm{NH}_{3}{ }^{\mathrm{a}}$} & $-57.3(0.5)$ & $365(47)$ & $5.1(0.7)$ & $-1.1(0.1)$ & $52(195)^{b}$ \\
\hline & $-61.6(0.6)$ & 201(43) & $5.9(1.1)$ & $4.8(0.8)$ & $8(8)$ \\
\hline \multirow[t]{2}{*}{$\mathrm{HNCO}^{\mathrm{c}}$} & $-58.9(0.0)$ & $195(0)$ & $4.0(0.1)$ & $0.1(-0.5)$ & $52(0)$ \\
\hline & $-63.9(10.0)$ & $205(50)$ & $4.0(1.0)$ & $0.9(-1.6)$ & $16(10)$ \\
\hline \multicolumn{6}{|c|}{ IRS 1 (Knez et al. 2009) } \\
\hline \multirow[t]{2}{*}{$\mathrm{C}_{2} \mathrm{H}_{2}$} & $-55.7(0.3)$ & $225(20)$ & $0.6(0.1)$ & $3.0(0.6)$ & $6(0.6)$ \\
\hline & $-59.4(0.3)$ & 191(10) & $0.6(0.1)$ & $2.8(0.2)$ & $24(1)$ \\
\hline \multirow[t]{2}{*}{$\mathrm{HCN}$} & $-56.3(0.3)$ & $256(30)$ & $0.8(0.1)$ & $5.6(2.8)$ & $8(1)$ \\
\hline & $-60.0(0.3)$ & $456(127)$ & $1.0(0.4)$ & $1.3(1.2)$ & $68(50)$ \\
\hline \multirow[t]{2}{*}{$\mathrm{NH}_{3}$} & $-57.3(0.3)$ & $278(31)$ & $0.9(1.0)$ & $5.2(1.5)$ & $9(1)$ \\
\hline & $-60.1(0.3)$ & $248(32)$ & $0.4(0.1)$ & $2.8(0.8)$ & $16(4)$ \\
\hline \multirow[t]{2}{*}{$\mathrm{HNCO}$} & $-57.2(0.3)$ & $319(27)$ & $1.8(0.2)$ & $0.4(0.3)$ & $4(0.3)$ \\
\hline & $-60.2(0.2)$ & 171(79) & $0.7(0.4)$ & $0.1(0.3)$ & $97(11)$ \\
\hline
\end{tabular}

Notes. Quantities in parentheses are the $1 \sigma$ uncertainties on the adjacent figures in the same units unless otherwise indicated.

${ }^{a}$ Negative column densities indicate components in emission. Column densities for the emission components are $C_{\mathrm{em}} \tau_{\mathrm{em}}(\nu)$ converted to $N$ and do not represent a real column densities.

${ }^{\mathrm{b}}$ Unable to be constrained in the fit, assuming lines are optically thin.

${ }^{c}$ All HNCO parameters other than the column density were fixed equal to those of $\mathrm{C}_{2} \mathrm{H}_{2}$ because an insufficient number of HNCO lines were observed to constrain them.

Table 4

$3 \sigma$ Upper Limits to Column Density of Molecules in the Upper State for Selected Non-detections

\begin{tabular}{lccc}
\hline \hline Species/Mode & Line & $n^{\mathrm{a}}$ & $\begin{array}{c}N_{\text {limit }}(\mathrm{X}) \\
\left(\mathrm{cm}^{-2}\right)\end{array}$ \\
\hline${ }^{13} \mathrm{CH}_{4} v_{4}{ }^{\mathrm{b}}$ & $R(1)$ & 1 & $4.1 \times 10^{16}$ \\
$\mathrm{OCS} v_{2}$ & $R(4)$ & 4 & $3.1 \times 10^{16}$ \\
$\mathrm{HCN} v_{2}$ & $P$ branch & 34 & $2.9 \times 10^{15}$ \\
& $R(15)$ & 1 & $1.8 \times 10^{16}$ \\
& $R(22)$ & 1 & $8.5 \times 10^{16}$ \\
$\mathrm{C}_{2} \mathrm{H}_{2} v_{5}$ & $R(23)$ & 1 & $1.1 \times 10^{17}$ \\
& $R(12)$ & 1 & $8.5 \times 10^{15}$ \\
$\mathrm{C}_{2} \mathrm{H}_{2} 2 v_{5}-v_{5}$ & $R(21)$ & 1 & $9.4 \times 10^{15}$ \\
& $R(22)$ & 1 & $3.5 \times 10^{16}$ \\
& $Q$ branch & 15 & $2.1 \times 10^{17}$
\end{tabular}

Notes. A uniform temperature of $200 \mathrm{~K}$ was assumed for all species.

a Number of lines/components in the attempted fit.

b The given values of $N\left({ }^{13} \mathrm{CH}_{4}\right)$ are for the isotopologue only rather than the total $\mathrm{CH}_{4}$ column density.

$10^{4} \mathrm{~K}$ ) is the critical density in the two-level approximation, using the values in Osterbrock \& Ferland (2006). The hydrogen recombination rate per unit volume is given by $n_{\text {recomb }}=\alpha_{B} n_{e} n_{p}$ where $\alpha_{B}\left(=2.6 \times 10^{-13} \mathrm{~cm}^{3} \mathrm{~s}^{-1}\right)$ is the recombination rate for "case $\mathrm{B}$ " recombination and $n_{p}$ is the proton density. The ratio of $N_{\text {Lyc }}$ to the [Ne II] luminosity is then given by

$$
\frac{N_{\mathrm{Lyc}}}{L_{[\mathrm{Ne} I \mathrm{I}}}=\frac{n_{\mathrm{recomb}}}{j_{[\mathrm{Ne} \mathrm{II}]}}=\frac{n_{\mathrm{H}^{+}}}{n_{\mathrm{Ne}^{+}}} \frac{\alpha_{B}}{q_{l u} h v}
$$

assuming $n_{e} \ll n_{c}$. The $\mathrm{Ne}^{+} / \mathrm{H}^{+}$ratio is given by the product of the $\mathrm{Ne}$ abundance and the singly ionized fraction for $\mathrm{Ne}, f_{+}$. We take the value of $f_{+}=0.3$ from Figure 3 in Ho \& Keto (2007) using for the radiation temperature that of a ZAMS star with IRS 9's luminosity $\left(T_{r}=26200 \mathrm{~K}\right)$ and a ratio of the dilution factor for blackbody radiation, $\Gamma$, to the electron density of $\Gamma / n_{e}=$ $10^{-19} \mathrm{~cm}^{3}$. From this we adopt an $\mathrm{Ne}^{+} / \mathrm{H}^{+}$ratio to be $3 \times 10^{-5}$.

Without knowing the width of the [Ne II] line, it is difficult to place a strong upper limit on its flux, but an emission equivalent width of $W_{\nu}=0.01 \mathrm{~cm}^{-1}$ can be ruled out unless the line is wider than $0.2 \mathrm{~cm}^{-1}$ or $77 \mathrm{~km} \mathrm{~s}^{-1}$. This corresponds to a line flux limit of $F_{[\mathrm{Ne} \mathrm{II}]}<2.0 \times 10^{-13} \mathrm{erg} \mathrm{s}^{-1} \mathrm{~cm}^{-2}$ and a luminosity limit of $L_{\text {[Ne II] }}=4 \pi d^{2} F_{\text {[Ne II] }}<1.7 \times 10^{32} \mathrm{erg} \mathrm{s}^{-1}$. We then calculate $N_{\text {Lyc }}<\left[3.3 \times 10^{4} \times 2.6 \times 10^{-13} / 8.4 \times 10^{-22}\right] \times 1.7 \times 10^{32}=$ $1.7 \times 10^{45}$ photons s $^{-1}$. Our result compares well with the radio continuum measurements of Sánchez-Monge et al. (2008), who find an ionizing flux of $0.9-2.4 \times 10^{45} \mathrm{~s}^{-1}$ towards IRS 9 and estimate a spectral type of B1. For reference, Tielens (2005) gives the Lyman continuum luminosity of an MS B0 star as $N_{\text {Lyc }}=1.4 \times 10^{48}$ photons s $^{-1}$. Apparently IRS 9 emits a small fraction of the Lyman continuum luminosity of an MS star of its bolometric luminosity. The radiation environment of IRS 1 is considerably more intense, by comparison. From infrared and millimeter data, the exciting source is inferred to be of spectral type earlier then O7.5, total luminosity $L>8 \times 10^{4} L_{\odot}$, and Lyman continuum luminosity $N_{\mathrm{Lyc}}>10^{48} \mathrm{~s}^{-1}$ (Hackwell et al. 1982; Akabane et al. 2001; Lugo et al. 2004). The inferred radiation field of IRS 1 is more intense than that of IRS 9 and results in a different set of conditions under which chemistry proceeds.

\section{INTERPRETATION OF THE SPECTROSCOPIC RESULTS}

\subsection{Assumptions and Caveats}

Interpretation of our results requires caution, given the underlying assumptions of the radiative transfer model in FITSPEC. 
The program assumes a single continuum source behind all absorbing material and a constant temperature characterizing the material in each of up to four absorbing "clouds" along the line of sight. We also add an optically thin emitting cloud, but neglect re-emission by the absorbing gas and absorption by the emitting gas. The column densities we report do not represent the entire column down to the embedded luminosity source, but rather the column only to an effective "dust photosphere" interior to which the continuum optical depth is large at a given wavelength. Consequently, our observations at $5 \mu \mathrm{m}$ and $8 \mu \mathrm{m}$ likely probe to greater depth than those at $13 \mu \mathrm{m}$. The optical depth may be higher at longer wavelengths due to silicate opacity, and the colder, outer layers of the dusty region are likely too cool to emit significantly at $5 \mu \mathrm{m}$. Comparison of results at different wavelengths is therefore difficult. We also did not take into account emission by molecules following absorption of photons, except as approximated by our "negative absorption" method of treating emission lines. Furthermore, we ignored emission following radiative excitation. We assumed either pure absorption or optically thin emission. However, the neglect of emission from a radiatively excited molecule should not be valid; at any plausible gas density the collisional de-excitation rate should be far below the radiative rate. Critical densities for vibrational transitions are typically greater than $10^{12} \mathrm{~cm}^{-3}$, and almost all absorption events should be followed by emission, not collisional de-excitation. For two of our molecules, $\mathrm{NH}_{3}$ and $\mathrm{C}_{2} \mathrm{H}_{2}$, we were able to observe lines of more than one vibrational band or rotational branch, and the comparison of the observed line depths provides evidence that re-emission following absorption is an important effect.

For $\mathrm{NH}_{3}$, we observed weak emission in $P$-branch lines at wavelengths near $12 \mu \mathrm{m}$ and possibly weak absorption in $Q$-branch lines near $10.5 \mu \mathrm{m}$. $R$-branch lines were not observed due to the silicate and telluric $\mathrm{O}_{3}$ absorption at their wavelengths. We explain the differences between the $P$ - and $Q$-branch lines based on the differing continuum radiation fields due to their differing proximity to the silicate dust feature. The $\mathrm{NH}_{3} R$ branch is centered on the $9.7 \mu \mathrm{m}$ silicate feature, the $Q$ branch falls on its long wavelength shoulder, and the $P$ branch is mostly between the 9.7 and $18 \mu \mathrm{m}$ features. If the radiation field seen by a molecule is due to silicate emission either because the molecule is exposed to radiation from an optically thin external dust cloud or because it is immersed in a cloud of warm silicate dust, there will be more upward radiative transitions (i.e., more absorption) in the $Q$ and $R$ branches than in the $P$ branch. The branching ratio between $P$-, $Q$-, and $R$-branch transitions from a given vibrationally excited state depends on the $K$ rotational quantum number, but is similar in the three bands. Consequently, if all absorption events are followed by an emission, absorptions will outnumber emissions in the $R$ branch and emissions will outnumbers absorptions in the $P$ branch, resulting in net emission in the $P$-branch lines, as we observe. In Section 5.3, we describe a simple model involving purely radiative transitions in $\mathrm{NH}_{3}$ incorporating upward and downward line strengths and assuming that the incident radiation field is proportional to the strength of the silicate feature at the wavelengths of the various lines. The model is time independent and assumes that the $\mathrm{NH}_{3}$ gas is optically thin. More detailed and quantitative models will follow in a future paper.

\section{2. $\mathrm{C}_{2} \mathrm{H}_{2}, \mathrm{HCN}, \mathrm{HNCO}, \mathrm{OCS}$, and $\mathrm{CO}$}

We find that excitation temperatures for various molecules are basically consistent toward IRS 9 and IRS 1 with the exception
Table 5

Abundances in IRS 9 and IRS 1 with Respect to $\mathrm{CO}$ and $\mathrm{H}_{2}$

\begin{tabular}{lccccc}
\hline \hline Molecule & \multicolumn{2}{c}{ IRS 9 (This Work) } & & \multicolumn{2}{c}{ IRS 1 (Knez et al. 2009) } \\
\cline { 2 - 3 } \cline { 5 - 6 } & $N(\mathrm{X}) / N(\mathrm{CO})$ & $N(\mathrm{X}) / N\left(\mathrm{H}_{2}\right)$ & & $N(\mathrm{X}) / N(\mathrm{CO})$ & $N(\mathrm{X}) / N\left(\mathrm{H}_{2}\right)$ \\
\hline $\mathrm{C}_{2} \mathrm{H}_{2}$ & $7.7(-3)$ & $3.3(-7)$ & & $5.7(-3)$ & $7.7(-7)$ \\
$\mathrm{HCN}$ & $1.4(-2)$ & $5.8(-7)$ & & $6.9(-3)$ & $9.2(-7)$ \\
$\mathrm{NH}_{3}$ & $1.9(-2)$ & $7.9(-7)$ & & $3.6(-2)$ & $4.8(-6)$ \\
$\mathrm{CH}_{4}$ & $9.9(-2)$ & $4.2(-6)$ & & $3.6(-2)$ & $4.8(-6)$ \\
$\mathrm{HNCO}$ & $<3.0(-3)$ & $<1.3(-7)$ & & $5.0(-4)$ & $6.0(-6)$ \\
\hline
\end{tabular}

Notes. All values are given in the notation $A(B)=A \times 10^{B}$. The column densities toward IRS 1 adopted for $\mathrm{CO}$ and $\mathrm{H}_{2}$ were $N\left({ }^{12} \mathrm{CO}\right)=1 \times 10^{19} \mathrm{~cm}^{-2}$ (Knez et al. 2009) and $N\left(\mathrm{H}_{2}\right)=7.5 \times 10^{22} \mathrm{~cm}^{-2}$ (Willner et al. 1982). For IRS 9 the ${ }^{12} \mathrm{CO}$ column density used was $N\left({ }^{12} \mathrm{CO}\right)=3.2 \times 10^{18} \mathrm{~cm}^{-2}$ (Boogert et al. 2002) and the $\mathrm{H}_{2}$ column density was the same as that used by Knez et al. (2009).

of the hotter of the two $\mathrm{NH}_{3}$ components toward IRS 1. That value of $T_{\mathrm{ex}}$ has a rather large uncertainty so it may in fact lie closer to $\sim 250 \mathrm{~K}$. The column densities are also similar in the two sources, though again with particular exception in the case of HCN. Knez et al. (2009) had the benefit of more observed HCN lines, but we find a lower column toward IRS 9. Most striking is the disagreement between the two sources in terms of the intrinsic line widths; although the thermal broadening should be similar, turbulence or systematic velocity variations along the line of sight to IRS 9 are greater than toward IRS 1. At the least, we can say that the spectral lines observed in the direction of IRS 9 are generally less saturated than those seen toward IRS 1 , and this has important consequences for the accuracy of the IRS 9 column densities we obtain.

An example in which saturation of lines becomes important is CO. Knez et al. (2009) derived fractional abundances relative to $\mathrm{CO}$ for a variety of molecules seen in absorption toward IRS 1 , attempting to compensate for badly saturated ${ }^{12} \mathrm{CO}$ lines by using the value of $N\left({ }^{13} \mathrm{CO}\right)$ in Mitchell et al. (1990) and adopting ${ }^{12} \mathrm{C} /{ }^{13} \mathrm{C}=45$ on the basis of their observations of $\mathrm{C}_{2} \mathrm{H}_{2}$. The value of $N\left({ }^{13} \mathrm{CO}\right)$ for IRS 9 reported by Mitchell et al. was based on their measurement of ${ }^{12} \mathrm{CO}$ lines and assumed the terrestrial ratio ${ }^{12} \mathrm{C} /{ }^{13} \mathrm{C}=89$. Boogert et al. (2004) confirm the saturation of the ${ }^{12} \mathrm{CO}$ lines observed toward IRS 9. From observations of lines of ${ }^{13} \mathrm{CO}$ and ${ }^{12} \mathrm{C}^{18} \mathrm{O}$ in upper $J$ levels of $7-15$ they derive an optical depth $\tau_{12}=13 \pm 4$ assuming ${ }^{12} \mathrm{C} /{ }^{13} \mathrm{C}=80$ from Boogert et al. (2002). However, they argue that reliable values for $N\left({ }^{12} \mathrm{CO}\right)$ can be obtained, since the ${ }^{13} \mathrm{CO}$ lines remain optically thin; they quote a value $N\left({ }^{12} \mathrm{CO}\right)=(3.2 \pm 1.0) \times 10^{18} \mathrm{~cm}^{-2}$. While we may argue with their choice of ${ }^{12} \mathrm{C} /{ }^{13} \mathrm{C}$ and with their conclusion that the ${ }^{13} \mathrm{CO}$ lines are optically thin, their value of the $\mathrm{CO}$ column density is probably more realistic for IRS 9 than that obtained from the lower-resolution observations of Mitchell et al. (1990). Abundance ratios for some of the molecules we observed with TEXES, relative to this column of CO, are shown in Table 5.

Similarly, an estimate of the abundance of various molecules with respect to $\mathrm{H}_{2}$ can be made given a measurement of the quantity of dust along the line of sight and a value for the gas-todust ratio in a typical protostellar envelope. Knez et al. adopted an $\mathrm{H}_{2}$ column density toward IRS 1 of $7.5 \times 10^{22} \mathrm{~cm}^{-2}$ based on the $9.7 \mu \mathrm{m}$ optical depth measured by Willner et al. (1982) to calculate fractional abundances of other molecules. Using the same method, we derive $N\left(\mathrm{H}_{2}\right)=5.2 \times 10^{22} \mathrm{~cm}^{-2}$ toward IRS 9 and present the resulting abundances in Table 5. The pattern of relative abundances differs between the objects according 
to both the molecular species and whether the abundance of a given species is referred to $\mathrm{CO}$ or to $\mathrm{H}_{2}$. The latter observation indicates that either the abundance ratio of $\mathrm{CO}$ to $\mathrm{H}_{2}$ or the gas-to-dust ratio varies between objects.

The assumptions made in calculating the values in Table 5-that the CO column density is comparable along both lines of sight and that the dust distribution and gas-to-dust ratio are similar in both cases-may not be unreasonable given that both objects are along nearby sightlines toward the same molecular cloud. Given this caveat, it is worth noting that the fractional abundances of all molecules relative to both $\mathrm{CO}$ and $\mathrm{H}_{2}$ are broadly consistent with each other, except for $\mathrm{HNCO}$ with the values given for IRS 9 properly unconstrained by FITSPEC because no $\mathrm{HNCO}$ lines were detected. $\mathrm{C}_{2} \mathrm{H}_{2}, \mathrm{HCN}$, and $\mathrm{NH}_{3}$ are generally less abundant in IRS 9 than IRS 1, although this is only a suggestion in light of the assumptions.

Some kinematic information about IRS 9 is available in the line shapes. In combination with the varying effective depths to which sightlines probe at different wavelengths, line shapes give a sense of the distribution of various molecules along the line of sight. The P Cygni line profiles of our CO observations, for example, clearly indicate an outflow known previously. $\mathrm{CH}_{4}$ absorption toward IRS 9 was known previously as well, but the TEXES data reveal the structure of the lines in more detail than the Keck+NIRSPEC spectra of Boogert et al. (2004) and the IRTF+Irshell spectra of Lacy et al. (1991).

In contrast to the circumstellar disk model of IRS 1 presented in Knez et al. (2009) based on TEXES observations, we cannot conclusively establish the presence of a disk in IRS 9. However, our fitting program preferred relatively small covering factors, similar to those in the best-fit synthetic spectra for the lower velocity component of IRS 1 . Knez et al. interpret this as the signature of a near edge-on disk, acting as a source of continuum radiation that is only partially covered by the absorbing material. Given the high-velocity outflow in IRS 9 reported by Mitchell \& Hasegawa (1991), we might rather be observing an accreting system nearly face-on in which the outflow both sweeps out a region near the continuum source and entrains material into the flow that only partially covers the source. This picture would account for the methane observations of Boogert et al. (2004) in which cold, solid-phase methane is seen in the outer envelope, becomes depleted in the outflow, and is seen in the gas phase in the inner envelope, all along a single sightline. We develop this model further in Section 5.6.

Non-detections of molecules also furnish some interpretable information. Carbonyl sulfide (OCS) is a molecule that commonly occurs in ices along sightlines through molecular clouds and indicates the presence of sulfur-bearing precursor species such as $\mathrm{H}_{2} \mathrm{~S}$ and $\mathrm{SO}_{2}$ (Ferrante et al. 2008). Gibb et al. (2004) detected solid-phase OCS toward IRS 9 with $I S O$, finding a column density of $(5.5 \pm 4.4) \times 10^{15} \mathrm{~cm}^{-2}$, and Knez et al. (2009) speculate that gas-phase OCS should be observable toward IRS 9 as indicated in some chemical models. We find an upper limit of $N(\mathrm{OCS}) \leqslant 2.9 \times 10^{15} \mathrm{~cm}^{-2}$ in the gas phase, which is compatible with the Gibb et al. result to within errors; we therefore conclude that any OCS near IRS 9 is firmly sequestered as an ice. Tielens (2005) cites an OCS ice abundance of $0.1 \%$ relative to $\mathrm{H}_{2} \mathrm{O}$ ice toward IRS 9 given a water ice column density of $1 \times 10^{19} \mathrm{~cm}^{-2}$. That figure may be compared to a CO ice abundance of $10 \%$. In interstellar ices, OCS is commonly found in association with hydrogenated species such as $\mathrm{H}_{2} \mathrm{O}$ and $\mathrm{CH}_{3} \mathrm{OH}$ (Palumbo et al. 1997; Dartois et al. 1999). Laboratory results reported by Collings et al. (2004) indicate that $\mathrm{OCS}$ in an $\mathrm{H}_{2} \mathrm{O}$ mixture desorbs at $\sim 150 \mathrm{~K}$, implying that significant quantities of this molecule may be frozen out in cold envelopes. However, $\mathrm{C}_{2} \mathrm{H}_{2}$ is seen toward both IRS 1 and IRS 9, for example, and according to the same study desorbs at only a slightly lower temperature. The relative columns of OCS and $\mathrm{C}_{2} \mathrm{H}_{2}$ may then reflect real differences in their abundances rather than their desorption temperatures. Alternately, OCS might only be abundant in the colder, outer envelope of IRS 9, explaining its apparent absence in the gas phase. Knez et al. (2009) did not observe OCS in the gas phase toward IRS 1 and Gibb et al. (2004) only cite an upper limit of $1 \times 10^{15} \mathrm{~cm}^{-2}$ for the column density in the solid phase. Other sulfur-bearing molecules desorb from grains at a relatively low temperature of $\sim 70 \mathrm{~K}$, well below the temperatures in both objects. It is difficult, then, to understand why high- $J$ lines of CS were observed toward IRS 1 by Knez et al. (2009) when recent models by Wakelam et al. (2011) underpredict the abundance of CS relative to other S-bearing molecules at temperatures above $100 \mathrm{~K}$. The IRS 1 CS gas-phase column density is comparable to the solid-phase column density of OCS toward IRS 9, so if IRS 1 began with a comparable OCS abundance it may indicate a reaction route preference for forming $\mathrm{CS}$ as opposed to, e.g., $\mathrm{SO}_{2}$. However, Wakelam et al. (2004) do not associate OCS and CS but rather assume that CS exists in the gas of the pre-evaporative phase. We did not search for CS or $\mathrm{SO}_{2}$ toward IRS 9 on the basis of their presumed low abundances, and thus are unable to comment on the likelihood of any OCS/CS association.

The occurrence of isocyanic acid (HNCO) may be tied to the presence of the cyanate ion $\left(\mathrm{OCN}^{-}\right)$, where $\mathrm{OCN}^{-}$is formed on grains by irradiation of solid HNCO in ices by UV in the presence of $\mathrm{NH}_{3}$ (van Broekhuizen et al. 2004). Once evaporated from grains, $\mathrm{OCN}^{-}$may then undergo hydrogenation to become $\mathrm{HNCO}$ again. We speculate, however, that the hydrogenation of $\mathrm{OCN}^{-}$takes place before desorption, since the charge an ion induces while on a grain would result in a relatively high desorption temperature. Knez et al. (2009) observed dozens of HNCO lines toward IRS 1 and infer a column density of $5.4 \times 10^{15} \mathrm{~cm}^{-2}$; this and the value of other gas parameters from their spectra led them to conclude that gas-phase HNCO may originate from evaporation of grain mantles by way of $\mathrm{OCN}^{-}$. A low column density compared to the value expected from the observed abundance of $\mathrm{OCN}^{-}$is interpreted as evidence that only a fraction of the available $\mathrm{OCN}^{-}$desorbed from grains becomes $\mathrm{HNCO}$, the remainder forming other molecules in gas-phase chemistry. Interpretation of observations is difficult, however, as Knez et al. point out that most chemical models do not currently include HNCO in their networks. All of the HNCO lines in the various TEXES settings we observed were non-detections. Our fitting program preferred column densities of $(0.1 \pm 0.5) \times$ $10^{16} \mathrm{~cm}^{-2}$ and $(0.9 \pm 1.6) \times 10^{16}$ in the two presumed absorbing units, although a clear lack of detected lines makes these values limits at best. We cannot, therefore, confidently distinguish between scenarios in which HNCO has a very low abundance in all phases and those in which it is effectively locked up in ices and unavailable to spectroscopy at our wavelengths.

\subsection{Radiative Excitation and Radiative Transfer}

In order to explain the observed pattern of $\mathrm{NH}_{3}$ absorption and emission in our spectra of IRS 9, we invoke a radiative transfer effect that emerged from our modeling efforts. We begin with gas in a shell of thickness $t$ around the continuum source with an $\mathrm{NH}_{3}$ density $n_{\mathrm{NH}_{3}}$. The rotational levels $(J, K)$ of the ground vibrational state are assumed to be populated thermally 
at temperature $T$. Electric dipole transitions to and from an upper vibrational level proceed according to the selection rule that $\Delta K=0, \Delta J= \pm 1$ or 0 . In ammonia there is also a doubling of each vibrational level into symmetric and antisymmetric $(s, a)$ inversion states, and the requirement that $s \leftrightarrow a$. Transitions are labeled by the inversion symmetry of the lower level. This gives the permitted transitions $a / s R\left(J_{u}-1, K\right), a / s Q\left(J_{u}, K\right)$, and $a / s$ $P\left(J_{u}+1, K\right)$. We obtain absorption coefficients $\alpha$ for each of these transitions from GEISA.

The absorption optical depth corresponding to $\alpha$ is

$$
\tau(w)=\alpha N\left(\mathrm{NH}_{3}\right) \phi_{w}
$$

in which $N\left(\mathrm{NH}_{3}\right)$ is the radial column density through the shell and $\phi_{w}$ is the line shape function, taken to be a Gaussian. Molecules are excited to the upper state along all branches at a rate

$$
r_{\text {up }}=\sum_{P, Q, R} \int \frac{L_{w}}{h c w} \tau(w) d w
$$

per second assuming $\tau \ll 1$ through the shell, where $L_{w}$ is the incident continuum luminosity. The rate at which photons are emitted on a given branch is determined by the rate of transitions populating the upper level and the branching ratio of the Einstein $A$ coefficients, e.g.,

$$
r_{\text {down }}(P)=r_{\text {up }}(P) \frac{A_{P}}{A_{P}+A_{Q}+A_{R}}
$$

for the $P$ branch. We calculate values for the $A$ coefficients according to

$$
A_{u l}=\frac{8 \pi c}{\lambda^{2}} \alpha(T) \frac{Q_{r}}{g_{u}} e^{E_{l} / k T},
$$

where $\lambda$ is the wavelength of the transition, $Q_{r}$ is the rotational partition function, $g_{u}$ is the statistical weight of the upper state, and $E_{l}$ is the energy of the lower state.

We consider the $a P(4, K), a Q(3, K)$, and $a R(2, K)$ lines that go to the $J=3$ rotational level of the $s v_{2}$ vibrational level. We use the branching ratios to compute the expected emission flux in a given line $l$ with wavenumber center $w$ from

$$
F_{l}=\frac{A_{l}}{A_{P}+A_{Q}+A_{R}} \frac{h c w}{4 \pi d^{2}} \sum_{P, Q, R} \frac{L_{w}}{h c w} N \alpha(T) .
$$

To calculate the net flux in a line we also need the amount of absorption. We require the relative intensity of the $10 \mu \mathrm{m}$ dust emission feature at the frequency of each of the branches in order to compute the absorption line flux. We use mid-infrared spectra of the young stars DI Cep and DK Tau obtained by Hanner et al. (1998) to which they fit models of optically thin silicate dust emission. From these spectra, we calculate relative intensities of $0.52,0.78$, and 1.00 for the continuum at the $11.7,10.7$, and $9.7 \mu \mathrm{m}$ wavelengths of the $a P, a Q$, and $a R$ lines for which $J_{u}=3$, respectively. The intensities are normalized to the value on the $a R$ branch as it is nearest to the peak of the $10 \mu \mathrm{m}$ silicate feature. The observed continuum flux near the $P$-branch lines, $\sim 60 \mathrm{Jy}$, is turned into a luminosity at the source by multiplying by $4 \pi d^{2}$, where $d$ is the distance to NGC $7538(2.65 \mathrm{kpc})$. We corrected for extinction using the $10 \mu \mathrm{m}$ optical depth measurement of Willner et al. (1982), which implies $A_{11.7 \mu \mathrm{m}} \sim 2.2 \mathrm{mag}$ and $A_{9.7 \mu \mathrm{m}} \sim 4.4 \mathrm{mag}$ toward IRS 9.

The extinction-corrected luminosity $\sim 1.5 \times 10^{35} \mathrm{erg}$ $\mathrm{s}^{-1} \mathrm{~cm}^{-1}$ at the position of the $P$-branch lines is scaled to the
Table 6

Predicted Fluxes for the $a P, a Q, a R$ Lines of $\nu_{2}$ Transitions of $\mathrm{NH}_{3}\left(J_{u}=3\right)$ and Measured Fluxes for the Corresponding a $P$-branch Lines from TEXES Spectra

\begin{tabular}{ccccc}
\hline \hline$K$ & \multicolumn{3}{c}{ Predicted } & Observed \\
\cline { 2 - 4 } & $\mathrm{F}_{a P}$ & $\mathrm{~F}_{a Q}$ & $\mathrm{~F}_{a R}$ & $\mathrm{~F}_{a P}$ \\
\hline 0 & 5.55 & $\ldots$ & -1.94 & 1.80 \\
1 & 2.64 & 0.03 & -0.95 & 1.35 \\
2 & 2.03 & 0.02 & -0.73 & $\ldots$ \\
3 & 2.11 & -0.87 & $\ldots$ & 0.90 \\
\hline
\end{tabular}

Notes. All fluxes are given in units of $10^{14} \mathrm{erg} \mathrm{s}^{-1} \mathrm{~cm}^{-2}$. Positive flux values indicate net emission while negative values indicate net absorption in a given line.

other branches according the values above. We assume throughout that the lines are sufficiently broad that they remain optically thin, leading to uncertainty in the covering factor. Only the product of column density and covering factor is meaningful under such circumstances, so we used the sum of this product for the two $\mathrm{NH}_{3}$ components rather than the sum of the $\mathrm{NH}_{3}$ column densities themselves in calculating $F_{l}$. We considered the possibility that the optically thin case might result in uncertainties propagating to the line fluxes, and to this end we carried out additional runs of FITSPEC in which we deliberately held the value of the covering factor fixed equal to 1 . This resulted in a variation of the product of column density and covering factor of $<20 \%$ compared to allowing the covering factor to vary. We therefore retained the assumption of the optically thin case, and opted to use the lower value for the product of column density and covering factor of $8.52 \times 10^{15} \mathrm{~cm}^{-2}$ in place of $N$ in Equation (17). For $\alpha(T)$, we chose an excitation temperature of $200 \mathrm{~K}$, consistent with the FITSPEC results for the $-61.6 \mathrm{~km} \mathrm{~s}^{-1}$ component. Finally, the assumed extinction was re-introduced at the end of the calculation to realistically predict the line fluxes observed at Earth.

The line fluxes we compute are given in Table 6 . The predicted fluxes are the differences of the strengths of the predicted emission and absorption components of each line and show an overall pattern of emission on the $P$ branch, weak absorption or no lines on the $Q$ branch, and absorption on the $R$ branch. The $P$-branch fluxes compare favorably with the integrated line fluxes from our data implying that our explanation is at least plausible. This suggests that the dust and $\mathrm{NH}_{3}$ are in close proximity to one another, if not actually mixed.

In the case of acetylene, we observed lines of the $\nu_{5} Q$ and $R$ branches at 12.5-13.7 $\mu \mathrm{m}$ and the $\nu_{4}+\nu_{5} P$ branch near $7.5 \mu \mathrm{m}$. The $v_{5} Q$ - and $v_{4}+v_{5} P$-branch lines are of low $\mathrm{S} / \mathrm{N}$, due to telluric interference, but clearly lead to higher $\mathrm{C}_{2} \mathrm{H}_{2}$ column densities than the $v_{5} R$-branch lines when the observations are interpreted with the pure absorption model. The $v_{4}+v_{5}$ lines appear to require at least an order of magnitude more $\mathrm{C}_{2} \mathrm{H}_{2}$ than do the $v_{5} \mathrm{R}$-branch lines. The radiative transfer effects discussed for $\mathrm{NH}_{3}$ may also be relevant for $\mathrm{C}_{2} \mathrm{H}_{2}$ and may explain a part of the discrepancy between the $\mathrm{C}_{2} \mathrm{H}_{2}$ bands. But the bands are farther from the silicate feature and the wavelength separation between the branches of the $\mathrm{C}_{2} \mathrm{H}_{2}$ bands is smaller than for $\mathrm{NH}_{3}$, so the effects should be smaller. The greater column density derived from the $v_{4}+v_{5}$ lines could instead be due to the smaller extinction at $7.5 \mu \mathrm{m}$ allowing a greater column of gas to be probed. This explanation was suggested by Evans et al. (1991) to explain a similar effect seen toward Orion IRc2, but it is difficult to understand how it could explain more than a factor 


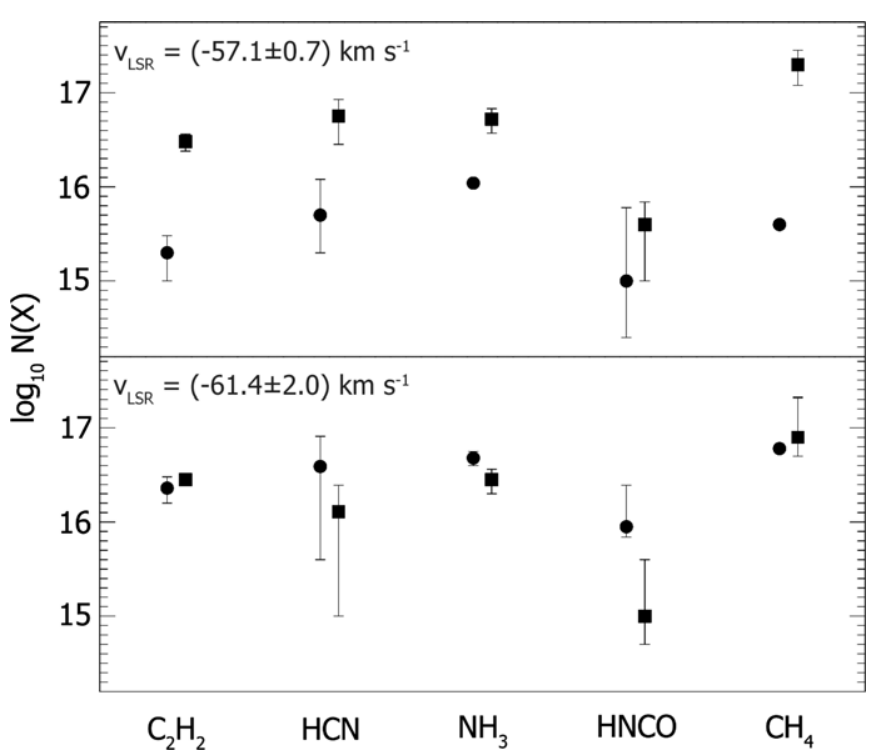

Figure 13. Log column densities for the main molecular species detected in this study for the two principal velocity components toward each target. IRS 9 values (this work) are shown as filled circles and IRS 1 values from Knez et al. (2009) as filled squares. The symbols for a given molecule are slightly offset for clarity.

$\sim 2$ discrepancy, given the ratio of extinction at the different wavelengths.

We propose a different explanation for the variation between the column densities derived from the $\mathrm{C}_{2} \mathrm{H}_{2} v_{5}$ and $v_{4}+v_{5}$ lines. Vibrational excitation caused by a $\nu_{5}$ band photon is almost always followed by emission of another $\nu_{5}$ band photon, although possibly in a different branch. (Due to the $\mathrm{C}_{2} \mathrm{H}_{2}$ selection rules, $P$ - or $R$-branch absorption can be followed by either $P$ - or $R$-branch emission, but $Q$-branch absorption must be followed by emission in the same branch.) However, $7.5 \mu \mathrm{m}$ $v_{4}+v_{5}$ absorption is most often followed by a $v_{4}+v_{5}-v_{4}$ transition at $\sim 13.7 \mu \mathrm{m}$ to the $\nu_{4}$ level. Since radiative decay from the $v_{4}$ level is forbidden, the $v_{4}$ excitation energy is then eventually lost in a collision. The result of this series of transitions is that there is little $v_{4}+v_{5}$ emission canceling the $v_{4}+v_{5}$ absorption, and these lines are probably reasonably well treated with the pure absorption model that we used. In contrast, the $v_{5}$ absorption lines may be largely canceled by emission, leading to an underestimate of the $\mathrm{C}_{2} \mathrm{H}_{2}$ column density. The amount of re-emission into our beam is very dependent on the (unknown) source geometry, which determines whether our line of sight has more or less than the average for the gas surrounding the source. Assuming the $v_{4}+v_{5}$ lines can be treated with a pure absorption model to give a fair measure of the abundance along our line of sight, the required correction to the column densities derived from the $v_{5}$ lines is approximately a factor of 10 . The required correction to the column densities of our other observed molecules may be similar.

\subsection{Abundance Trends and Relative Ages}

In Figure 13, we present a graphical comparison of the column densities of $\mathrm{C}_{2} \mathrm{H}_{2}, \mathrm{HCN}, \mathrm{NH}_{3}, \mathrm{HNCO}$, and $\mathrm{CH}_{4}$ we obtain toward IRS 9 with those derived for IRS 1 in Knez et al. (2009). Column densities for each of the two components with similar LSR velocities $\left(-57 \mathrm{~km} \mathrm{~s}^{-1}\right.$ and $\left.-61 \mathrm{~km} \mathrm{~s}^{-1}\right)$ toward each object are shown. We reach rather different conclusions for the two velocity components; IRS 1 appears to be clearly enhanced in all molecules except for HNCO at $-57 \mathrm{~km} \mathrm{~s}^{-1}$, whereas the column densities are essentially the same to within errors at $-61 \mathrm{~km} \mathrm{~s}^{-1}$. HNCO is the exception at the higher negative velocity, but we again stress that the cited values for IRS 9 are those preferred by our fitting program despite HNCO being an evident non-detection in our data. It is therefore not strictly reliable in comparison with the firm detection of HNCO toward IRS 1 . Also, we do not have any evidence a priori that these two velocity components are kinematically related. Knez et al. (2009) claim that the two components seen toward IRS 1 are real but may not be separate; they may instead represent a nonGaussian velocity distribution. If the latter situation is true, then such a velocity distribution may be systemic in other parts of NGC 7538. Regardless, the pattern that emerges from Figure 13 is suggestive of real differences in the gas-phase abundance of these molecules, which we attribute to differing thermal conditions in the environment of each object. If the molecular abundances in all phases were the same to begin with, the gasphase abundances we see at the present time are indicative of a difference in the relative ages of IRS 9 and IRS 1 .

Several other lines of evidence also argue in favor of a younger age for IRS 9. First, a number of molecules (e.g., $\mathrm{H}_{2} \mathrm{O}, \mathrm{CH}_{3} \mathrm{OH}$, $\mathrm{HCOOH}$, and $\mathrm{OCN}^{-}$) are seen in the solid phase in the spectrum of IRS 9 that are either in the gas phase or apparently absent toward IRS 1. $\mathrm{CO}$ and $\mathrm{CH}_{4}$ are seen both in the solid and gas phases, toward IRS 9 although the gas-phase lines probably form in the shocked outflow material. Second, a fairly strong ionizing radiation field exists near IRS 1 , whereas our non-detection of the $12.8 \mu \mathrm{m}$ line of [Ne II] discussed in Section 4.4 argues for a much weaker field toward IRS 9. In addition, IRS 1 has an ionized component to its outflow, probably driven by a wind; IRS 9 shows no similar outflow component. Third, the chemistry of IRS 1 resembles the "hot core" phase in which ices desorb from dust grains, leading to a short-lived $\left(\sim 10^{4}-10^{5} \mathrm{yr}\right)$ hightemperature gas-phase chemistry and the synthesis of complex organic molecular species (van der Tak 2005). Some species, such as HNCO, are clear detections toward IRS 1 but appear to be absent toward IRS 9; we cannot rule out an intrinsic abundance effect in explaining this difference, but a simpler explanation is that the IRS 9 core is not yet sufficiently warm to evaporate it.

Knez et al. (2009) claim an age of $\sim 10^{5}$ yr for IRS 1 based on their chemical models. However, there is significant uncertainty in that value. Abundances of the molecules they detected indicate an age closer to $\sim 10^{4} \mathrm{yr}$, but for other molecules the chemical model predictions disagree with the observed values, suggesting ages in the range of $2 \times 10^{3}$ to $2 \times$ $10^{6} \mathrm{yr}$. They explain this large range as a result of insufficiently detailed models. The models of Doty et al. (2002) predict enhancements in the abundances of $\mathrm{C}_{2} \mathrm{H}_{2}, \mathrm{HCN}$, and $\mathrm{CH}_{4}$ for temperatures above about $800 \mathrm{~K}$ at late times $\left(\geqslant 10^{5} \mathrm{yr}\right)$; the enhancements are similar to those observed toward IRS 1, but the temperatures derived by Knez et al. (2009) are considerably lower than this. The fractional abundance of HCN in the Doty et al. model for $200 \mathrm{~K}$ quickly turns down at times $>10^{5} \mathrm{yr}$ whereas those of $\mathrm{C}_{2} \mathrm{H}_{2}$ and $\mathrm{CH}_{4}$ increase through at least $10^{5.5} \mathrm{yr} ; N(\mathrm{HCN})$ is in fact slightly higher toward IRS 1 compared to IRS 9 by a factor of about 1.4. This may both support the argument that IRS 1 is the older object of the two and suggest an upper limit for its age, since the HCN abundance has not begun the predicted decline, but this conclusion is tentative since our value of $N(\mathrm{HCN})$ for IRS 9 is determined by the singular detection of the $v_{2} R(16)$ line. Lastly, an additional age constraint from chemistry is provided by the non-detection of 
the molecule CCS by Hoffman \& Seojin Kim (2011) in Green Bank Telescope (GBT) data, suggesting an age $\geqslant 10^{4} \mathrm{yr}$. Given that we find IRS 9 to be in an evidently more primitive chemical state than IRS 1 , the upper limit to its age is probably $\sim 10^{4} \mathrm{yr}$. Detailed, robust chemical modeling, using our observations as inputs, may help more firmly constrain this value.

Our results, when considered in the context of the earlier work on IRS 1, qualitatively support a picture of an evolutionary sequence of cores in NGC 7538 consistent with the models of Elmegreen \& Lada (1977) and Campbell \& Thompson (1984) in which a wave of shock/ionization fronts from a previous generation of stars caused the sequential collapse of the cores that became IRS 1 and IRS 9, respectively. As the wave continues to propagate toward the southeast, it should initiate the collapse of cores found in progressively more primitive states. The "IR-quiet" protostellar object NGC $7538 \mathrm{~S}$ is likely the next youngest object in the region after IRS 9 (Pestalozzi et al. 2006). Such a sequence, including NGC 7538 IRS 11 among the youngest objects, is supported by the maser observations of Hutawarakorn \& Cohen (2003). It is tempting to assume that the wave has left the IRS 1 region already, but several newly discovered submillimeter sources within $0.35 \mathrm{pc}$ of IRS 1 reported by Qiu et al. (2011), presumably harboring forming intermediate- or high-mass stars, may be younger than IRS 9 and contemporaneous with NGC 7538 S. However, they may also be fragments, along with IRS 1, of a common progenitor. The star formation history of NGC 7538 may therefore be more complex than can be represented with a simple, monotonically increasing series of protostellar ages.

\subsection{Spatial Resolution of IRS 9}

We checked whether IRS 9 is spatially resolved in TEXES data by obtaining spatial-spectral maps of IRS 9 and $\mu$ Cep, a mid-infrared- bright standard star that was used as a point spread function (PSF) reference for spatial deconvolution. To generate the maps, the TEXES slit was stepped across each object and spectral information gathered at each step, building up data cubes in which there are two spatial dimensions and one spectral dimension. Additional steps were included at both the beginning and end of the scans to sample the sky for removal. The data are handled by our reduction pipeline in a manner substantially similar to the method of processing the nodded observations, treating a scan as a series of two-dimensional spectrograms.

IRS 9 and $\mu$ Cep were observed with identical scan parameters including scan direction, length, and step size, and were observed at comparable zenith angles. After reducing the raw data, different spectral windows were selected from which to form reconstructed images of each object by summing the data in the spectral direction. For IRS 9, we chose a window centered at about $745.88 \mathrm{~cm}^{-1}$, near the position of the $\mathrm{C}_{2} \mathrm{H}_{2} \nu_{5} R(6)$ line in the rest frame of the object. The window was $0.35 \mathrm{~cm}^{-1}$ wide. We selected this frequency range to search for spatial extent in the IRS 9 map as we noted absorption in the $R(6)$ line in nod-mode spectra. For the deconvolution reference, we chose a nearby spectral region apparently free from telluric and stellar photospheric absorption centered at $744.43 \mathrm{~cm}^{-1}$ and $0.25 \mathrm{~cm}^{-1}$ in width. The observed FWHM for each object was 0 .'67 for IRS 9 and 0.'63 for $\mu$ Cep at each object's respective spectral setting. The deconvolution was performed using the maximum entropy method (Bryan \& Skilling 1980; Narayan \& Nityananda 1986), with results shown in Figure 14. The deconvolved image of IRS 9 in the $\mathrm{C}_{2} \mathrm{H}_{2}$ feature appears pointlike

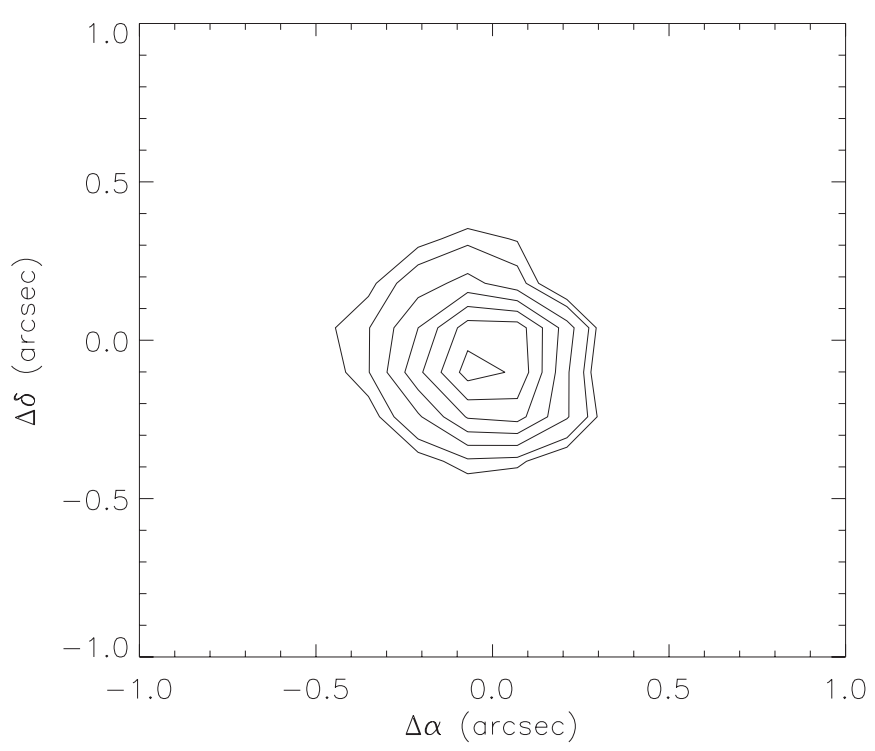

Figure 14. Contour plot of the TEXES scan map of NGC 7538 IRS 9 at $745.8 \mathrm{~cm}^{-1}$ after maximum entropy deconvolution. The PSF reference for the deconvolution was a scan map of the mid-infrared standard $\mu$ Cep made with the same parameters as the IRS 9 scan. North is up and east at left and contours are plotted at flux densities of 25, 50, 100, 150, 200, 250, and $300 \mathrm{Jy}$. The plot origin is approximately the location of the source peak intensity.

with some possible weak extension toward the NE. We do not believe this indicates that the envelope or other structure of IRS 9 is spatially resolved, and we carried out our other TEXES observations exclusively in nod mode.

This approach to imaging and deconvolution should be interpreted cautiously. Poor seeing during a scan, for example, might distort the PSF on timescales shorter than the time required to scan across an object. However, we felt sufficiently convinced that IRS 9 presents itself as a point source in the mid-infrared that we did no further scans. Given the distance to NGC 7538 and the spatial resolution of our observations, we conclude that spatial structure of IRS 9 is unresolved on scales $\leqslant 2000$ AU. This limit is consistent with the inferred sizes of disks detected around protostars with $10^{3}-10^{5} L_{\odot}$ (Patel et al. 2005; Schreyer et al. 2006; Rodríguez et al. 2007; Franco-Hernández et al. 2009; Galván-Madrid et al. 2010) and places a constraint on physical models of massive protostars in NGC 7538, which often include disks thought to be hundreds of AU in size (e.g., Sandell et al. 2009; Surcis et al. 2011).

\subsection{A Structural Model of IRS 9}

Based on the conclusions we reach from kinematic indications in the data, we compose a toy model of the IRS 9 system to show its essential structure and a guess at the viewing geometry. This model is shown in Figure 15. In this view, the central perhaps accreting object is shown in cross-section with a surrounding disk. The known bipolar outflow is indicated by the evacuated regions above and below the disk and by arrows showing the direction of the flow. A lighter shading above and below the disk represents the remaining envelope that has not been accreted or become part of the outflow. In order to account for seeing certain molecules under presumed LTE conditions while not observing the exciting source directly, we suggest a "dust photosphere" at a radius from the source at which the emission from the dust becomes optically thick, as indicated by dashed lines in the figure. It is this effective source of radiation against which we see most of our absorption lines. Since we do not resolve 


\section{A}

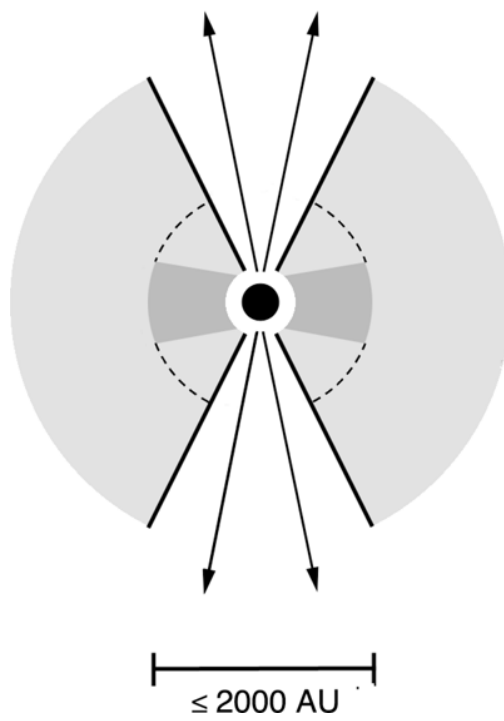

Figure 15. Schematic rendering of a proposed model of NGC 7538 IRS 9 based on TEXES data. A central, embedded source (or sources) has at least partially cleared cavities in a bipolar outflow while significant dust remains in a spherical distribution (light gray shading). An inner disk may exist (dark gray shading). To account for our observations, the line of sight samples both scattered light in the outflow as well as a "dust photosphere" (dashed lines) where the dust becomes opaque at a mid-infrared wavelengths. The inferred viewing angle is shown, and a scale bar is provided based on the constraints discussed in Section 5.5. Note that the envelope and outflow cavities as depicted are not shown to this scale.

spatial structure in the molecular absorption region above the dust photosphere, we interpret the size scale constraint discussed in the previous section (2000 AU) as an upper limit to the radius of the dust photosphere itself. This value also represents an upper limit of the size of the disk, because a larger, optically thick disk should have been detectable by virtue of its spatial extent. Our data do not permit a constraint to be placed on the size of the surrounding envelope or the outflow cavities.

The inclusion of a disk in this picture is tentative, given that the lifetime of disks around massive stars is thought to be particularly short, of order $10^{-4}$ the lifetime of the host star (Mann \& Williams 2009). The evolutionary state of IRS 9 may be sufficiently early in its history, well before the embedded protostar is visible, to retain a disk. Knez et al. (2009) argue for a disk around IRS 1 whose surface is observed at near-grazing incidence; a sightline through the disk atmosphere is proposed to explain their observations.

We propose a viewing angle based on the following. First, the broad CO lines with their large, Doppler-shifted velocity separations suggest formation in the bipolar outflow. Second, the apparent radiative "pumping" of the $\mathrm{NH}_{3}$ transitions, discussed in Section 5.3, requires continuum light from the direction of the dust photosphere. Third, the other molecular species we observed in absorption also require a background continuum source, though the range of excitation conditions we derive is insufficient to definitively locate them in the envelope or the turbulent edge of the outflow cavity. The $\mathrm{C}_{2} \mathrm{H}_{2}$ combination mode may be an exception and could shock-excited in the outflow. Consequently, we place the viewing angle such that the observer is looking nearly along the edge of the outflow cavity and sees light from both sources. This model appears to account for all aspects of our observations in at least a qualitative way. It is also consistent with the conclusion of Sandell et al. (2005), based on $\mathrm{HCO}^{+} J=2 \rightarrow 1$ observations showing deep, redshifted self-absorption, that the molecular outflow is viewed nearly pole-on.

\section{SUMMARY}

We have presented the method and results of a study of the embedded high-mass protostellar object NGC 7538 IRS 9 and compared our findings to a similar investigation of the related object NGC 7538 IRS 1. We obtained high-resolution, midinfrared spectra of these objects in 46 ro-vibrational transitions of the fundamental bands of the molecules $\mathrm{C}_{2} \mathrm{H}_{2}, \mathrm{CH}_{4}, \mathrm{HCN}$, $\mathrm{NH}_{3}$, and $\mathrm{CO}$ and a number of their isotopologues. We also detected two lines of the $v_{4}+v_{5}$ combination mode of $\mathrm{C}_{2} \mathrm{H}_{2}$. From these observations, we draw some broad conclusions.

1. IRS 9 appears to be a spatially unresolved object on a scale of $\sim 2000 \mathrm{AU}$.

2. With the exception of $\mathrm{CO}$, whose lines are saturated, we did not observe ${ }^{13} \mathrm{C}$ isotopologues of any organic molecules. This may indicate a particularly large value of the ${ }^{12} \mathrm{C} /{ }^{13} \mathrm{C}$ ratio, or the ${ }^{13} \mathrm{C}$ isotopologue lines were simply below our threshold of detection.

3. There is no discernible, consistent trend in the abundance variations of $\mathrm{C}_{2} \mathrm{H}_{2}, \mathrm{HCN}, \mathrm{NH}_{3}$, and $\mathrm{CH}_{4}$ with respect to either $\mathrm{CO}$ or $\mathrm{H}_{2}$ between IRS 9 and IRS 1. Column density variations between the two objects are an order of magnitude or less in each case.

4. The observation of gas-phase CS absorption toward IRS 1 and the evident non-detection of sulfur-bearing species like OCS in the gas phase toward IRS 9 may reflect a real $\mathrm{S}$ abundance variation between the two objects but can also be explained by different thermal conditions in each case. A similar abundance discrepancy involving $\mathrm{HNCO}$ may indicate sufficiently low temperatures in the IRS 9 envelope to retain it as an ice and explain its detection in the solid phase by Gibb et al. (2004). If the abundances in all phases are actually comparable between the two sources, a relatively weak heating source is implied in the case of IRS 9. While this is at odds with the somewhat higher inferred abundances of the other molecules toward the warmer environment of IRS 1, the suggested sightline in that direction through a disk atmosphere subjects those molecules to direct irradiation by protostellar UV. This may drive photochemistry that lowers abundances of certain molecules as they are converted to other kinds.

5. However, the relative abundances of various molecular species with respect to $\mathrm{CO}$ and $\mathrm{H}_{2}$ can also be explained by variations in the $\mathrm{CO} / \mathrm{H}_{2}$ ratio or the gas-to-dust ratio between the two objects. Choosing between these interpretations would require better determining the $\mathrm{CO}$ and $\mathrm{H}_{2}$ abundances toward both objects.

6. Lines of many species observed toward IRS 9 have higher Doppler $b$ values than those toward IRS 1 , resulting in lines toward IRS 9 being less saturated than those toward IRS 1 .

7. Given the non-detection of $\mathrm{Ne}$ II in our data and the continuum flux density at the expected wavenumber of the line (70 $\pm 10 \mathrm{Jy})$, we find a corresponding upper limit of $\sim 10^{45}$ photons $\mathrm{s}^{-1}$ for the Lyman continuum flux in IRS 9. This value is well below both the expected flux of a ZAMS star with the same luminosity and the ionizing flux of IRS $1\left(>10^{48}\right.$ photons $\left.\mathrm{s}^{-1}\right)$.

8. The observed pattern of emission and absorption components of ammonia lines in our spectra can be explained by 
a non-LTE radiative transfer effect involving "pumping" of lines on the $R$-branch by light from the $9.7 \mu \mathrm{m}$ silicate dust feature. Predictions of the strength of this effect compare reasonably well with observed fluxes in $P$-branch lines.

9. Our data are consistent with a simple model of the IRS 9 system in which the sightline probes both a high-speed outflow and a quiescent envelope illuminated from behind by a "dust photosphere" such that line formation depth is wavelength dependent. The possibility of seeing the turbulent flow along the wall of the outflow cavity is supported by the broad, blueshifted wing of the $\mathrm{C}_{2} \mathrm{H}_{2} v_{4}+v_{5}$ combination band that may arise in a shock. A disk is included in the model given the system's inferred young age and the presence of a kinematically indicated disk toward the (presumably) older IRS 1.

10. Observed differences in the abundance of various molecular species toward IRS 9 and IRS 1 imply an upper limit to the age of IRS 9 of $\sim 10^{4} \mathrm{yr}$, whereas IRS 1 may be an order of magnitude older. This conclusion is consistent with the triggered star formation models of Elmegreen \& Lada (1977) as applied to NGC 7538 by Campbell \& Thompson (1984) and others.

The authors thank Marty Bitner, Dan Jaffe, Tommy Greathouse, and Matt Richter for assistance in obtaining the observations presented here. We recognize the efforts of the support staff at both the IRTF and Gemini who helped make our observing runs productive and efficient. We also gratefully acknowledge the constructive comments and suggestions of an anonymous referee, which substantially improved the manuscript before publication. The data presented here are based in part on observations obtained at the Gemini Observatory, which is operated by the Association of Universities for Research in Astronomy, Inc., under a cooperative agreement with the NSF on behalf of the Gemini partnership: the National Science Foundation (United States), the Science and Technology Facilities Council (United Kingdom), the National Research Council (Canada), CONICYT (Chile), the Australian Research Council (Australia), CNPq (Brazil), and SECYT (Argentina). Additional data were obtained at the Infrared Telescope Facility, which is operated by the University of Hawaii under Cooperative Agreement No. NNX08AE38A with the National Aeronautics and Space Administration, Science Mission Directorate, Planetary Astronomy Program. J.C.B. and J.H.L. acknowledge the support of the National Science Foundation (NSF) through grant AST-0607312.

\section{REFERENCES}

Akabane, K., Matsuo, H., Kuno, N., \& Sugitani, K. 2001, PASJ, 53, 821

Araya, E., Hofner, P., Goss, W. M., et al. 2007, ApJS, 170, 152

Arnett, D. (ed.) 1996, Supernovae and Nucleosynthesis: An Investigation of the History of Matter from the Big Bang to the Present (Princeton, NJ: Princeton Univ. Press)

Bally, J., \& Zinnecker, H. 2005, AJ, 129, 2281

Balog, Z., Kenyon, S. J., Lada, E. A., et al. 2004, AJ, 128, 2942

Beichman, C. A., Neugebauer, G., Habing, H. J., Clegg, P. E., \& Chester, T. J. (ed.) 1988, Infrared Astronomical Satellite (IRAS) Catalogs and Atlases, Explanatory Supplement, Vol. 1 (Washington, DC: GPO)

Beuther, H., Linz, H., \& Henning, T. 2012, A\&A, 543, A88

Bevington, P. R., \& Robinson, D. K. (ed.) 2003, Data Reduction and Error Analysis for the Physical Sciences (3rd ed.; Boston, MA: McGraw-Hill)

Blaauw, A. 1964, ARA\&A, 2, 213

Blaauw, A. 1991, in NATO ASIC Proc. 342: The Physics of Star Formation and Early Stellar Evolution, ed. C. J. Lada \& N. D. Kylafis (Kluwer: Dordrecht), 125
Bonnell, I. A., \& Bate, M. R. 2005, MNRAS, 362, 915

Bonnell, I. A., Bate, M. R., Clarke, C. J., \& Pringle, J. E. 2001, MNRAS, 323, 785

Bonnell, I. A., Bate, M. R., \& Zinnecker, H. 1998, MNRAS, 298, 93

Bonnell, I. A., Vine, S. G., \& Bate, M. R. 2004, MNRAS, 349, 735

Boogert, A. C. A., Blake, G. A., \& Öberg, K. 2004, ApJ, 615, 344

Boogert, A. C. A., Blake, G. A., \& Tielens, A. G. G. M. 2002, ApJ, 577,271

Brunt, C. M. 2003, ApJ, 584, 293

Bryan, R. K., \& Skilling, J. 1980, MNRAS, 191, 69

Campbell, B., \& Thompson, R. I. 1984, ApJ, 279, 650

Collings, M. P., Anderson, M. A., Chen, R., et al. 2004, MNRAS, 354, 1133

Dartois, E., Demyk, K., d'Hendecourt, L., \& Ehrenfreund, P. 1999, A\&A, 351, 1066

Dickel, H. R., Dickel, J. R., \& Wilson, W. J. 1981, ApJ, 250, L43

Doty, S. D., Schöier, F. L., \& van Dishoeck, E. F. 2004, A\&A, 418, 1021

Doty, S. D., van Dishoeck, E. F., van der Tak, F. F. S., \& Boonman, A. M. S. 2002, A\&A, 389, 446

Ekström, S., Georgy, C., Eggenberger, P., et al. 2012, A\&A, 537, A146

Elmegreen, B. G., \& Lada, C. J. 1977, ApJ, 214, 725

Evans, N. J., II, Lacy, J. H., \& Carr, J. S. 1991, ApJ, 383, 674

Ferrante, R. F., Moore, M. H., Spiliotis, M. M., \& Hudson, R. L. 2008, ApJ, 684,1210

Fischer, J., Joyce, R. R., Righini-Cohen, G., Simon, M., \& Simon, T. 1980, ApJ, 240, L95

Foster, T., \& Routledge, D. 2003, ApJ, 598, 1005

Franco-Hernández, R., Moran, J. M., Rodríguez, L. F., \& Garay, G. 2009, ApJ, 701,974

Galván-Madrid, R., Zhang, Q., Keto, E., et al. 2010, ApJ, 725, 17

Gibb, E. L., Whittet, D. C. B., Boogert, A. C. A., \& Tielens, A. G. G. M. 2004, ApJS, 151, 35

Hackwell, J. A., Grasdalen, G. L., \& Gehrz, R. D. 1982, ApJ, 252, 250

Hanner, M. S., Brooke, T. Y., \& Tokunaga, A. T. 1998, ApJ, 502, 871

Henkel, C., Wilson, T. L., \& Johnston, K. J. 1984, ApJ, 282, L93

Heyer, M. H., Carpenter, J. M., \& Snell, R. L. 2001, ApJ, 551, 852

Ho, L. C., \& Keto, E. 2007, ApJ, 658, 314

Hoffman, I. M., \& Seojin Kim, S. 2011, AJ, 142, 202

Hutawarakorn, B., \& Cohen, R. J. 2003, MNRAS, 345, 175

Jacquinet-Husson, N., Scott, N. A., Chédin, A., et al. 2005, J. Quant. Spectrosc. Radiat. Transfer, 95, 429

Jiang, Z., Tamura, M., Hoare, M. G., et al. 2008, ApJ, 673, L175

Johnston, K. G., Keto, E., Robitaille, T. P., \& Wood, K. 2011, MNRAS, 415 , 2953

Kameya, O. 2006, in Proc. 8th European VLBI Network Symp. (Trieste: SISSA)

Keto, E., \& Zhang, Q. 2010, MNRAS, 406, 102

Knez, C., Lacy, J. H., Evans, N. J., van Dishoeck, E. F., \& Richter, M. J. 2009, ApJ, 696, 471

Lacy, J. H., Carr, J. S., Evans, N. J., II, et al. 1991, ApJ, 376, 556

Lacy, J. H., \& Jaffe, D. T. 2007, in 211 Meeting of American Astronomical Society, BAAS, 39, 866

Lacy, J. H., Richter, M. J., Greathouse, T. K., Jaffe, D. T., \& Zhu, Q. 2002, PASP, 114,153

Lugo, J., Lizano, S., \& Garay, G. 2004, ApJ, 614, 807

Mann, R. K., \& Williams, J. P. 2009, ApJ, 694, L36

McKee, C. F., \& Ostriker, E. C. 2007, ARA\&A, 45, 565

Michelson, A. A. 1898, ApJ, 8, 37

Mitchell, G. F., \& Hasegawa, T. I. 1991, ApJ, 371, L33

Mitchell, G. F., Maillard, J.-P., Allen, M., Beer, R., \& Belcourt, K. 1990, ApJ, 363,554

Moscadelli, L., Reid, M. J., Menten, K. M., et al. 2009, ApJ, 693, 406

Narayan, R., \& Nityananda, R. 1986, ARA\&A, 24, 127

Osterbrock, D. E., \& Ferland, G. J. (ed.) 2006, Astrophysics of Gaseous Nebulae and Active Galactic Nuclei (2nd ed.; Sausalito, CA: Univ. Science Books)

Palumbo, M. E., Geballe, T. R., \& Tielens, A. G. G. M. 1997, ApJ, 479, 839

Panagia, N. 1973, AJ, 78, 929

Patel, N. A., Curiel, S., Sridharan, T. K., et al. 2005, Nature, 437, 109

Pendleton, Y. J., Tielens, A. G. G. M., Tokunaga, A. T., \& Bernstein, M. P. 1999, ApJ, 513, 294

Pestalozzi, M. R., Elitzur, M., Conway, J. E., \& Booth, R. S. 2004, ApJ, 603, L113

Pestalozzi, M. R., Minier, V., Motte, F., \& Conway, J. E. 2006, A\&A, 448, L57

Qiu, K., Zhang, Q., \& Menten, K. M. 2011, ApJ, 728, 6

Reid, M. A., \& Wilson, C. D. 2005, ApJ, 625, 891

Rodríguez, L. F., Zapata, L. A., \& Ho, P. T. P. 2007, ApJ, 654, L143

Sánchez-Monge, Á., Palau, A., Estrella, R., Beltrán, M. T., \& Girart, J. M. 2008, A\&A, 485, 497 
Sandell, G., Goss, W. M., \& Wright, M. 2005, ApJ, 621, 839

Sandell, G., Goss, W. M., Wright, M., \& Corder, S. 2009, ApJ, 699, L31

Schreyer, K., Semenov, D., Henning, T., \& Forbrich, J. 2006, ApJ, 637, L129

Smith, R. L., Pontoppidan, K. M., Young, E. D., Morris, M. R., \& van Dishoeck, E. F. 2009, ApJ, 701, 163

Surcis, G., Vlemmings, W. H. T., Torres, R. M., van Langevelde, H. J., \& Hutawarakorn Kramer, B. 2011, A\&A, 533, A47

Tielens, A. G. G. M. (ed.) 2005, The Physics and Chemistry of the Interstellar Medium (Cambridge, UK: Cambridge Univ. Press)

Ulich, B. L., \& Haas, R. W. 1976, ApJS, 30, 247

van Broekhuizen, F. A., Keane, J. V., \& Schutte, W. A. 2004, A\&A, 415, 425 van der Tak, F. F. S. 2005, in IAU Symp. 227, Massive Star Birth: A Crossroads of Astrophysics, ed. R. Cesaroni, M. Felli, E. Churchwell, \& M. Walmsley (Cambridge: Cambridge Univ. Press), 70

Wakelam, V., Caselli, P., Ceccarelli, C., Herbst, E., \& Castets, A. 2004, A\&A, 422, 159

Wakelam, V., Hersant, F., \& Herpin, F. 2011, A\&A, 529, A112

Werner, M. W., Becklin, E. E., Gatley, I., et al. 1979, MNRAS, 188, 463

Whittet, D. C. B., Schutte, W. A., Tielens, A. G. G. M., et al. 1996, A\&A, 315, L357

Willner, S. P., Gillett, F. C., Herter, T. L., et al. 1982, ApJ, 253, 174

Wilson, T. L. 1999, Rep. Prog. Phys., 62, 143

Zinnecker, H., \& Yorke, H. W. 2007, ARA\&A, 45, 481 